IGPG-06/11-4, AEI-2006-086

gr-qc/0611112

\title{
Effective constraints of loop quantum gravity
}

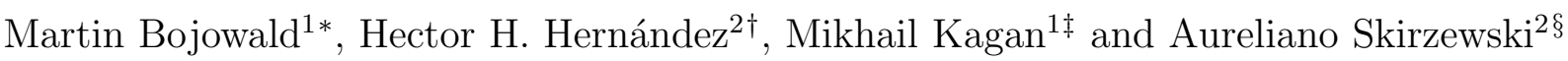 \\ ${ }^{1}$ Institute for Gravitational Physics and Geometry, The Pennsylvania State University, \\ 104 Davey Lab, University Park, PA 16802, USA \\ ${ }^{2}$ Max-Planck-Institut für Gravitationsphysik, Albert-Einstein-Institut, \\ Am Mühlenberg 1, D-14476 Potsdam, Germany
}

\begin{abstract}
Within a perturbative cosmological regime of loop quantum gravity corrections to effective constraints are computed. This takes into account all inhomogeneous degrees of freedom relevant for scalar metric modes around flat space and results in explicit expressions for modified coefficients and of higher order terms. It also illustrates the role of different scales determining the relative magnitude of corrections. Our results demonstrate that loop quantum gravity has the correct classical limit, at least in its sector of cosmological perturbations around flat space, in the sense of perturbative effective theory.
\end{abstract}

\section{Introduction}

Interacting quantum theories in low energy or semiclassical regimes can be described by effective equations which amend the classical ones by correction terms. Compared to the full quantum description an analysis of such effective equations is much easier once they have been derived. In addition to the simpler mathematical structure, difficult conceptual and interpretational problems of wave functions can be evaded, still allowing one to compute potentially observable effects. Technical and conceptual problems are even more severe in quantum gravity, in particular in background independent formulations. Yet, especially in this case observational guidance would be of invaluable help. Since the high energy regimes of cosmology are commonly considered as the best access to such guidance, an effective description for fields relevant for early universe cosmology is needed. In this paper we use an effective framework of perturbative loop quantum gravity around a spatially flat isotropic background space-time to derive correction terms to the classical constraints.

\footnotetext{
*e-mail address: bojowald@gravity.psu.edu

$\dagger$ e-mail address: hehe@aei.mpg.de

${ }^{\ddagger}$ e-mail address: mak411@psu . edu

${ }^{\S}$ e-mail address: skirz@aei.mpg.de
} 
Our analysis will be done for scalar metric modes in longitudinal gauge as this can be used to simplify the perturbative basic variables. They can then be chosen to be of diagonal form, although now fully inhomogeneous, which is the main reason for simplifications as they have been used extensively in symmetric models [1, 2, 3, 4]. The main constructions of these models can thus be extended, in a similarly explicit form, to inhomogeneous situations without assuming any symmetry. This allows us to compute explicit corrections to effective constraints which, in combination with the Hamiltonian analysis of cosmological perturbation theory in [5], leads to corrected perturbation equations and new effects [6].

A physical regime is selected by introducing a background geometry in the background independent quantization through a specific class of states [7]. This keeps the characteristic background independent features of the quantum theory, such as its spatial discreteness, intact while bringing the theory to a form suitable for perturbative expansions and applications. In the perturbative regime, we will make use of special structures provided by the geometrical background which can usually be chosen to allow symmetries, e.g. isotropy for cosmological perturbations around a Friedmann-Robertson-Walker model. In particular, we use this to introduce regular lattice states with a spacing (in geometrical variables rather than embedding coordinates) whose size determines at which scales quantum effects become important. The geometrical spacing thus specifies on which scales physical fields are being probed by a given class of states. On such a lattice, explicit calculations can be done.

We demonstrate this by providing higher curvature terms as well as corrections to inverse powers of metric components. Several issues that arose in isotropic models will be clarified. Finally, we discuss general aspects of effective equations and the semiclassical limit of loop quantum gravity. The article thus consists of two parts, an explicit scheme to derive correction terms presented in Sec. 3 and 4, and a more general discussion of effective equations and the classical limit in Sec. 5 .

\section{Basic variables and operators}

The basic variables of interest for a canonical formulation of gravity [8] are the spatial metric $q_{a b}$ occurring in the space-time metric

$$
\mathrm{d} s^{2}=-N^{2} \mathrm{~d} t^{2}+q_{a b}\left(\mathrm{~d} x^{a}+N^{a} \mathrm{~d} t\right)\left(\mathrm{d} x^{b}+N^{b} \mathrm{~d} t\right) .
$$

(or equivalent quantities such as a triad $e_{a}^{i}$ with $e_{a}^{i} e_{b}^{i}=q_{a b}$ or its inverse $e_{i}^{a}$ ), extrinsic curvature $K_{a b}=(2 N)^{-1}\left(\dot{q}_{a b}-D_{a} N_{b}-D_{b} N_{a}\right)$ (or related objects such as the Ashtekar connection) and matter fields with their momenta. The components $N$ (lapse function) and $N^{a}$ (shift vector) of the space-time metric are not dynamical, and thus do not have momenta, but are important for selecting the space-time slicing or the gauge.

Because of their role in loop quantum gravity, we will use Ashtekar variables [9, 10] which are a densitized triad $E_{i}^{a}=\left|\operatorname{det} e_{b}^{j}\right| e_{i}^{a}$ and the connection $A_{a}^{i}=\Gamma_{a}^{i}-\gamma K_{a}^{i}$ with the spin connection

$$
\Gamma_{a}^{i}=-\epsilon^{i j k} e_{j}^{b}\left(\partial_{[a} e_{b]}^{k}+\frac{1}{2} e_{k}^{c} e_{a}^{l} \partial_{[c} e_{b]}^{l}\right)
$$


compatible with the triad, $K_{a}^{i}=K_{a b} e_{i}^{b}$ and the positive Barbero-Immirzi parameter $\gamma$ [10, 11. We use them here in perturbative form on a flat isotropic metric background, focusing on scalar modes. This means, as explained in more detail in [5], that the unperturbed metric as well as its perturbations can be assumed to be diagonal, $E_{i}^{a}=\tilde{p}^{(i)}(x) \delta_{i}^{a}$, which simplifies calculations. For scalar modes, all diagonal components of the metric $q_{a b}=$ $a^{2}\left(1-2 \psi(x)^{2}\right) \delta_{a b}$ are in fact equal, but we will see that this restriction is not general enough for formulating a loop quantization. Moreover, we can choose a vanishing shift vector $N^{a}=0$, implying that extrinsic curvature $K_{a}^{i}=\tilde{k}_{(i)}(x) \delta_{a}^{i}$ is diagonal, too. (The Ashtekar connection, on the other hand, will not be diagonal because it has non-diagonal contributions from the spin connection. It is of the form $A_{a}^{i}=\tilde{k}_{(i)}(x) \delta_{a}^{i}+\psi_{I}(x) \epsilon_{a}^{i I}$ where the non-diagonal part $\psi_{I}$ arising from the spin connection computed in Sec. 3.1.4 can be dealt with perturbatively.) Our calculations will thus be done in a given gauge, and would be more complicated in others. Nevertheless, we are including the general perturbations of metric and matter variables relevant for scalar modes without too strong restrictions as they could arise in other gauges.

\subsection{Gauge choices and their implications for a quantization}

In general, the space-time gauge is determined by prescribing the behavior of lapse function $N$ and shift vector $N^{a}$ occurring in a metric (1). The lapse function, as we will see, can be chosen arbitrarily in our calculations, but the shift vector is restricted for a diagonal perturbation to be realized. We are thus using a particular class of gauges in setting up our calculations, although we do not explicitly make use of the form of gauge transformations. This is important because the canonical constraints, most importantly the Hamiltonian constraint $H$ in addition to the diffeomorphism constraint $D_{a}$, and thus also the gauge transformations $\delta_{\xi} f=\left\{f, \xi^{0} H+\xi^{a} D_{a}\right\}$ they generate will be corrected by quantum effects. Classical properties of the gauge transformations should thus not be used before one computes quantum corrections. It is then a priori unclear which particular gauge choices, other than fixing lapse and shift directly, are allowed. Some gauges implicitly refer to gauge transformation equations to relate metric to matter perturbations, or to select the space-time slicing such as for the flat gauge. In this example, one would make use of gauge transformations to set the spatial metric perturbation equal to zero which allows one to focus calculations on the simpler matter part. In this process, one solves gauge transformation equations of the metric perturbation, depending on lapse and shift, such that the transformed perturbation vanishes. This determines a gauge to be chosen, but makes use of explicit gauge transformation equations which are not guaranteed to remain unchanged with quantum corrections. Our choice of vanishing shift, on the other hand, is harmless because it does not refer to explicit gauge transformation equations. We are thus working at a more general level keeping metric and matter perturbations independent. A combined gauge invariant combination of the two perturbations can be determined once the quantum corrected gauge transformations have been computed.

When constraints are modified, manifest covariance of the resulting equation becomes an issue as it is discussed in more detail in [5]. Such quantum corrections are derived from 
effective constraints of gravity which are defined as expectation values of quantum gravity operators in general states [12]. We motivate the procedure here briefly, and will provide some further information in Sec. 5, for details we refer to [12, 13, 14]. If constraint operators satisfy the classical constraint algebra, covariance would be manifest for the expectation values. But there is an additional step involved in deriving effective equations: the expectation values depend on infinitely many quantum variables such as the spreads of states which do not have classical analogs. Effective equations are obtained by truncating this to finitely many fields (similarly to the derivative expansion in low energy effective actions), resulting in equations of motion of the classical form corrected by quantum terms. Indeed, any quantum theory is based on states which are not just determined by expectation values of the basic variables such as $A_{a}^{i}$ and $E_{i}^{a}$ in loop quantum gravity. Expectation values of the basic variables would correspond to the classical values in constraint expressions, but there are infinitely many further parameters such as the spread and deformations of the state from a Gaussian. These additional, quantum variables can suitably be parameterized in the form

$$
G_{q}^{a, n}=\left\langle(\hat{q}-\langle\hat{q}\rangle)^{n-a}\left(\hat{p}_{q}-\left\langle\hat{p}_{q}\right\rangle\right)^{a}\right\rangle_{\text {Weyl }}
$$

for any degree of freedom $\left(q, p_{q}\right)$ present in the classical theory. Here, $1<n \in \mathbb{N}, 0 \leq a \leq n$, and the subscript "Weyl" denotes totally symmetric ordering. Every classical degree of freedom thus does not only give rise to expectation values $\langle\hat{q}\rangle$ and $\left\langle\hat{p}_{q}\right\rangle$ but to infinitely many additional quantum variables. All of these variables are dynamical, and are in general coupled to each other.

Moreover, expectation values of most operators, including Hamiltonians, in general states depend on all these infinitely many variables. This is to be reduced to a finite set for an effective description which introduces quantum correction terms into the classical equations. In particular, spread and deformation parameters are usually assumed to be subdominant compared to expectation values. Without explicitly constructing semiclassical states satisfying such conditions, one can make semiclassicality assumptions for those parameters to be negligible. This is what we will do in this paper as a shortcut to deriving effective expressions from a full quantum theory. Since special quantization steps are involved in the construction of operators which reformulate classical expressions, corrections in effective equations will result which are not sensitive to the precise form of semiclassical states.

\subsection{Lattice states and basic operators}

We are thus able to implement all degrees of freedom needed for inhomogeneities in a way which is accessible to explicit calculations. While the general kinematical arena of loop quantum gravity is based on discrete spatial structures built on arbitrary graphs with possibly high-valent vertices, we will use regular lattices with 6 -valent vertices. Regularity of the lattice is implemented by making use of symmetries of the background we are perturbing around: The three independent generators of translational symmetry define lattice directions. In explicit constructions of lattice states, a scale $\ell_{0}$ will appear which 
is the coordinate length of lattice links measured in a given, fixed embedding 1 But this parameter is independent of the quantum variables assigned to each link we will be using, which means that the quantum theory will be defined on "freely floating" lattices as in the full theory, respecting diffeomorphism invariance. The scale $\ell_{0}$ will only become important in the continuum limit, when making contact between the quantum variables and classical continuous fields. This obviously breaks manifest diffeomorphism covariance, just as the classical perturbation theory in basic fields rather than gauge-invariant combinations, since the classical perturbations are written with respect to a background space-time.

Compared to the full theory, we are restricting states by assuming regularity and thus allowing, e.g., only unknotted links and vertices of valence at most six. This turns out to be sufficient to include all relevant perturbative degrees of freedom. While the general graphs of loop quantum gravity allow more freedom, its meaning is not known and appears redundant in our application.

\subsubsection{Holonomies and fluxes}

The canonical fields are given by $\left(A_{a}^{i}, E_{j}^{b}\right)$ which are to be turned into operators on a suitable Hilbert space. To set this up, we need to choose a functional representation of state, which is conveniently done in the connection representation where states are functionals of $A_{a}^{i}$. According to loop quantum gravity, lattice graphs then label states and determine their expressions as functions of connections: a state associated with a given graph depends on the connection only through holonomies

$$
h_{e}(A)=\mathcal{P} \exp \left(\int_{e} \mathrm{~d} t A_{a}^{i} \dot{e}^{a} \tau_{i}\right)
$$

along its edges. Here $\tau_{j}=-\frac{i}{2} \sigma_{j}$ are the $S U(2)$-generators in terms of Pauli matrices $\sigma_{j}$ and $\mathcal{P}$ denotes path ordering. That those are the basic objects represented on a Hilbert space together with fluxes

$$
F_{S}(E)=\int_{S} \mathrm{~d}^{2} y E_{i}^{a} \tau^{i} n_{a}
$$

for surfaces $S$ with co-normal $n_{a}$ is the basic assumption of loop quantum gravity [15]. Our corrections to cosmological perturbation equations will be implications of this fact and thus test the theory directly. Using the perturbative form of $A_{a}^{i}$, we can split off perturbatively the non-diagonal part (composed of spin connection components) in an expansion and exploit the diagonality of the remaining part to obtain $h_{v, I}=\exp \left(\gamma \tau_{I} \int_{e_{v, I}} \mathrm{~d} t \tilde{k}_{I}\left(e_{v, I}(t)\right)\right.$. Similarly, fluxes will be of the form $F_{v, I}=\int_{S_{v, I}} \mathrm{~d}^{2} y \tilde{p}^{I}(y)$. A lattice link starting at a vertex $v$ in direction $I$ in a fixed orientation is denoted $e_{v, I}$, and a lattice plaquette transversal to this edge and centered at its midpoint as $S_{v, I}$. (Here and in the following set-up we closely follow [7] to which we refer the reader for more details; note, however, that $\gamma$ has been absorbed there in $\tilde{k}_{I}$.)

\footnotetext{
${ }^{1}$ The coordinate length need not be the same for all links, but can be chosen this way without loss of generality.
} 
Matrix elements of the variables $h_{v, I}$ together with $F_{v, I}$ form the basic objects of loop quantum gravity in this setting. They are thus elementary degrees of freedom, comparable to atoms in condensed matter. Classical fields will, as we display in detail later, emerge from these objects in suitable regimes and limits only. Even in such regimes where one can recover the usual metric perturbations there will in general be correction terms examples of which we aim to compute below. Correction functions will then also depend on the basic objects $h_{v, I}$ and $F_{v, I}$ directly, which can be expressed through the classical metric perturbations in a secondary step 2

To recover the correct semiclassical behavior one has to make sure that effective equations of motion can indeed be written in a form close to the classical ones. Since classical Hamiltonians are local functionals of extrinsic curvature and densitized triad components, it must then be possible to approximate the non-local, integrated objects $h_{v, I}$ and $F_{v, I}$ by local values of $\tilde{k}_{I}$ and $\tilde{p}^{I}$ evaluated in single points This is indeed possible if we assume that $\tilde{k}_{I}$ is approximately constant along any edge, whose coordinate lengths are $\ell_{0}=\int_{e_{v, I}} \mathrm{~d} t$. We can then write

$$
h_{v, I}=\exp \left(\int_{e_{v, I}} \mathrm{~d} t \gamma \tilde{k}_{I} \tau^{I}\right) \approx \cos \left(\ell_{0} \gamma \tilde{k}_{I}(v+I / 2) / 2\right)+2 \tau_{I} \sin \left(\ell_{0} \gamma \tilde{k}_{I}(v+I / 2) / 2\right)
$$

where $v+I / 2$ denotes, in a slight abuse of notation, the midpoint of the edge which we use as the most symmetric relation between holonomies and continuous fields, and

$$
F_{v, I}=\int_{S_{v, I}} \tilde{p}^{I}(y) \mathrm{d}^{2} y \approx \ell_{0}^{2} \tilde{p}^{I}(v+I / 2)
$$

(note that the surface $S_{v, I}$ is defined to be centered at the midpoint of the edge $e_{v, I}$ ). This requires the lattice to be fine enough, which will be true in regimes where fields are not strongly varying. For more general regimes this assumption has to be dropped and non-local objects appear even in effective approximations since a function $\tilde{k}_{I}$ will be underdetermined in terms of the $h_{v, I}$. Since the recovered classical fields must be continuous, this means that they can arise only if quantizations of $h_{v, I}$ and $F_{v, I}$, respectively, for nearby lattice links do not have too much differing expectation values in a semiclassical state. If this is not satisfied, continuous classical fields can only be recovered after a process of coarse graining as we will briefly discuss in Sec. 5.2.1.

In addition to the assumption of slowly varying fields on the lattice scale, we have also made use of the diagonality of extrinsic curvature which allows us to evaluate the holonomy in a simple way without taking care of the factor ordering of $\mathrm{su}(2)$-values along the path. We can thus re-formulate the theory in terms of U(1)-holonomies

$$
\eta_{v, I}=\exp \left(i \int_{e_{v, I}} \mathrm{~d} t \gamma \tilde{k}_{I} / 2\right) \approx \exp \left(i \ell_{0} \gamma \tilde{k}_{I}(v+I / 2) / 2\right)
$$

\footnotetext{
${ }^{2}$ This already indicates that difficulties which were sometimes perceived in isotropic models, where corrections seemed to depend on the scale factor whose total scale is undetermined, do not occur in this inhomogeneous setup.
} 
along all lattice links $e_{v, I}$. On the lattice, a basis of all possible states is then given by specifying an integer label $\mu_{v, I}$ for each edge starting at vertex $v$ in direction $I$ and defining

$$
\left\langle\tilde{k}(x) \mid \ldots, \mu_{v, I}, \ldots\right\rangle:=\prod_{v, I} \exp \left(i \mu_{v, I} \int_{e_{v, I}} \mathrm{~d} t \gamma \tilde{k}_{I} / 2\right)
$$

as the functional form of the state $\left|\ldots, \mu_{v, I}, \ldots\right\rangle$ in the $k$-representation. The form of the states is a consequence of the representation of holonomies. States are functions of U(1)holonomies, and any such function can be expanded in terms of irreducible representations which for $\mathrm{U}(1)$ are just integer powers. This would be more complicated if we allowed all possible, also non-diagonal, curvature components as one is doing in the full theory. In such a case, one would not be able to reduce the original $\mathrm{SU}(2)$-holonomies to simple phase factors and more complicated multiplication rules would have to be considered. In particular, one would have to make sure that matrix elements of holonomies are multiplied with each other in such a way that functions invariant under $\mathrm{SU}(2)$-gauge rotations result [16]. This requires additional vertex labels which we do not need in the perturbative situation.

For the same reason we have simple multiplication operators given by holonomies associated with lattice links,

$$
\hat{\eta}_{v, I}\left|\ldots, \mu_{v^{\prime}, J}, \ldots\right\rangle=\left|\ldots, \mu_{v, I}+1, \ldots\right\rangle .
$$

There are also derivative operators with respect to $\tilde{k}_{I}$, quantizing the conjugate triad components. Just as holonomies are obtained by integrating the connection or extrinsic curvature, densitized triad components are integrated on surfaces, then called fluxes (4), before they can be quantized. For a surface $S$ of lattice plaquette size intersecting a single edge $e_{v, I}$ outside a vertex, we have

$$
\hat{F}_{v, I}\left|\ldots, \mu_{v^{\prime}, J}, \ldots\right\rangle=4 \pi \gamma \ell_{\mathrm{P}}^{2} \mu_{v, I}\left|\ldots, \mu_{v^{\prime}, J}, \ldots\right\rangle
$$

or

$$
\hat{\mathcal{F}}_{v, I}\left|\ldots, \mu_{v^{\prime}, J}, \ldots\right\rangle=2 \pi \gamma \ell_{\mathrm{P}}^{2}\left(\mu_{v-I, I}+\mu_{v, I}\right)\left|\ldots, \mu_{v^{\prime}, J}, \ldots\right\rangle .
$$

if the intersection happens to be at the vertex. The Planck length $\ell_{\mathrm{P}}=\sqrt{G \hbar}$ arises through a combination of $G$ from the basic Poisson brackets and $\hbar$ from a quantization of momenta as derivative operators. Here, in a similar notation as above, $v-I$ denotes the vertex preceding $v$ along direction $I$ in the given orientation. We will later call such labels simply $\mu_{v-I, I}=\mu_{v,-I}$ as illustrated in Fig. 1. These operators quantize integrated triad components (6). This shows that all basic degrees of freedom relevant for us can be implemented without having to use the more involved SU(2)-formulation.

Note, that even for scalar perturbations which classically have triads proportional to the identity, distinct $\tilde{p}^{I}(v)$-components have to be treated differently at the quantum level. One cannot assume all edge labels around any given vertex to be identical while still allowing inhomogeneity. Moreover, operators require local edge holonomies which change one edge label $\mu_{v, I}$ independently of the others. Similarly, corresponding operators $\hat{F}_{v, I}$ 


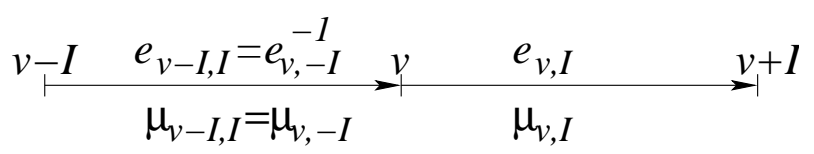

Figure 1: Edges adjacent to a vertex $v$ in a given direction $I$. For the edge oriented oppositely to the chosen one for direction $I$, the labels " $v-I, I$ " and " $v,-I$ " can be chosen interchangeably, defining in this way negative values for the label $I$.

and $\hat{F}_{v, J}(I \neq J)$ act on different links coming out of a vertex $v$ and have thus independent eigenvalues in general. To pick a regime of scalar modes, one will choose a state whose edge fluxes are peaked close to the same triad value in all directions and whose holonomies are peaked close to the same exponentiated extrinsic curvature values, thus giving effective equations for a single scalar mode function. But this to equal values in different directions cannot be done at the level of operators.

These basic operators $h_{v, I}$ and $F_{v, I}$ will appear in more complicated ones and in particular in the constraints. As we can see, they depend not directly on the classical fields $\tilde{p}^{I}(x)$ and $\tilde{k}_{I}(x)$ but, in local approximations, on quantities $p^{I}(x):=\ell_{0}^{2} \tilde{p}^{I}(x)$ and $k_{I}(x):=\ell_{0} \tilde{k}_{I}(x)$ rescaled by factors of the lattice link size $\ell_{0}$. This re-scaling occurring automatically in our basic variables has two advantages: It makes the basic variables independent of coordinates and provides them unambiguously with dimensions of length squared for $p$ while $k$ becomes dimensionless. (Otherwise, one could choose to put dimensions in coordinates or in metric components which would make arguments for the expected relevance of quantum corrections more complicated.) This also happens in homogeneous models [17], but in that case, especially in spatially flat models, there was sometimes confusion about the meaning of even the re-scaled variables. This is because the scale factor, for instance, as the isotropic analog of $\tilde{p}^{I}$ could be multiplied by an arbitrary constant and thus the total scale would have no meaning even when multiplied by the analog of $\ell_{0}^{2}$. Thus, correction functions depending on this quantity in an isotropic model require an additional assumption on how the total scale is fixed.

This is not necessary in inhomogeneous situations. Here, the quantities $p^{I}$ will appear in quantum corrections and their values determine unambiguously when corrections become important. The corresponding fluxes are the relevant quantum excitations, and when they are close to the Planck scale quantum corrections will unambiguously become large. On the other hand, if the $p^{I}$ become too large, approaching the Hubble length squared or a typical wave length squared, discreteness effects become noticeable even in usual physics. As we will see in more detail in Sec. 5.2.2, this allows one to estimate orders of magnitudes of corrections to be expected even without detailed calculations [6]. Although the size of the $p^{I}$ is coordinate independent, unlike the value of the scale factor, say, its relation to the classical field depends on $\ell_{0}$ and thus on the lattice size. It may thus appear that $p^{I}$ is coordinate dependent, but this is clearly not the case because it derives directly from a coordinate independent flux. The lattice values are defined independently of coordinates, just by attaching labels $\mu_{v, I}$ to lattice links. Once they have been specified and the lattice has been embedded in a spatial manifold, their relation to classical metric 
fields can be determined. It is, of course, the classical fields such as metric components which depend on the coordinate choice when they are tensorial. The relation between $p^{I}$ and the classical metric depends on the lattice spacing measured in coordinates because the representation of the classical metric itself depends on which coordinates have been chosen. Thus, our basic quantities are coordinate independent and coordinates enter only when classical descriptions are recovered in a semiclassical limit.

\subsubsection{Volume}

An important ingredient to construct constraints is the volume operator. Using the classical

expression $V=\int \mathrm{d}^{3} x \sqrt{\left|\tilde{p}^{1} \tilde{p}^{2} \tilde{p}^{3}\right|}$ we introduce the volume operator $\hat{V}=\sum_{v} \prod_{I=1}^{3} \sqrt{\left|\hat{\mathcal{F}}_{v, I}\right|}$ which, using (11), has eigenvalues

$$
V\left(\left\{\mu_{v, I}\right\}\right)=\left(2 \pi \gamma \ell_{\mathrm{P}}^{2}\right)^{3 / 2} \sum_{v} \prod_{I=1}^{3} \sqrt{\left|\mu_{v, I}+\mu_{v-I, I}\right|} .
$$

While densitized triad components are directly implemented through basic fluxes, the process of quantizing triad or co-triad components is more indirect. While they are uniquely determined from the densitized triad classically, one needs to take inverse components. With flux operators having discrete spectra containing zero, this is not possible in a direct manner at the quantum level. Nevertheless [18], one can construct operators for co-triad components based on the classical identity

$$
\left\{A_{a}^{i}, \int \sqrt{|\operatorname{det} E|} \mathrm{d}^{3} x\right\}=2 \pi \gamma G \epsilon^{i j k} \epsilon_{a b c} \frac{E_{j}^{b} E_{k}^{c}}{\sqrt{|\operatorname{det} E|}}=4 \pi \gamma G e_{a}^{i} .
$$

On the left hand side, no inverse appears and we just need to express connection components in terms of holonomies, use the volume operator and replace the Poisson bracket by a commutator. Resulting operators are then of the form $h_{e}\left[h_{e}^{-1}, \hat{V}\right]$ for $\mathrm{SU}(2)$-holonomies along suitable edges $e$, e.g.

$$
\operatorname{tr}\left(\tau^{i} h_{v, I}\left[h_{v, I}^{-1}, \hat{V}_{v}\right]\right) \sim-\frac{1}{2} i \hbar \ell_{0}\left\{\widehat{A_{a}^{i}, V_{v}}\right\}
$$

for $h_{v, I}$ as in (5). This shows that factors of the link size $\ell_{0}$ are needed in reformulating Poisson brackets through commutators with holonomies, which, as will become clear below, are provided by the discretized integration measure in spatial integrations such as they occur in the Hamiltonian constraint.

\section{Hamiltonian constraint}

Holonomies, the volume operator and commutators between them are finally used to define Hamiltonian constraint operators. We will briefly describe the general procedure and then derive resulting correction terms in effective equations for both gravitational and matter contributions to the constraint. 


\subsection{Gravitational part}

The gravitational contribution to the Hamiltonian constraint is given by

$$
\begin{aligned}
H[N]= & \frac{1}{16 \pi G} \int_{\Sigma} \mathrm{d}^{3} x N|\operatorname{det} E|^{-1 / 2}\left(\epsilon_{i j k} F_{a b}^{i} E_{j}^{a} E_{k}^{b}\right. \\
& \left.-2\left(1+\gamma^{-2}\right)\left(A_{a}^{i}-\Gamma_{a}^{i}\right)\left(A_{b}^{j}-\Gamma_{b}^{j}\right) E_{i}^{[a} E_{j}^{b]}\right)
\end{aligned}
$$

in terms of Ashtekar variables with the curvature $F_{a b}^{i}=2 \partial_{[a} A_{b]}^{i}+\epsilon^{i j k} A_{a}^{j} A_{b}^{k}$. The second term, quadratic in extrinsic curvature components $K_{a}^{i}=\gamma^{-1}\left(\Gamma_{a}^{i}-A_{a}^{i}\right)$, is in general more complicated to deal with due to the appearance of spin connection components as functionals of $E_{i}^{a}$ through (2). One usually starts with quantizing the first term and then uses the identity [18]

$$
K_{a}^{i}=\gamma^{-1}\left(A_{a}^{i}-\Gamma_{a}^{i}\right) \propto\left\{A_{a}^{i},\left\{\int \mathrm{d}^{3} x F_{a b}^{i} \frac{\epsilon^{i j k} E_{j}^{a} E_{k}^{b}}{\sqrt{|\operatorname{det} E|}}, \int \sqrt{|\operatorname{det} E|} \mathrm{d}^{3} x\right\}\right\}
$$

which allows one to express the second contribution in terms of the first. In the first term, then, the densitized triad components including the inverse determinant can be quantized using (13), and the curvature components $F_{a b}^{i}$ can be obtained through holonomies around appropriately chosen small loops [19]. On our regular lattices, natural loops based at a given vertex are provided by the adjacent lattice plaquettes. After replacing the Poisson brackets by commutators, the resulting first part of the Hamiltonian operator, $\hat{H}^{(1)}=\sum_{v} \hat{H}_{v}^{(1)}$ has non-zero action only in vertices of a lattice state, each contribution being of the form

$$
\begin{aligned}
\hat{H}_{v}^{(1)}= & \frac{1}{16 \pi G} \frac{2 i}{8 \pi \gamma G \hbar} \frac{N(v)}{8} \sum_{I J K} \sum_{\sigma_{I} \in\{ \pm 1\}} \sigma_{1} \sigma_{2} \sigma_{3} \epsilon^{I J K} \\
& \times \operatorname{tr}\left(h_{v, \sigma_{I} I}(A) h_{v+\sigma_{I} I, \sigma_{J} J}(A) h_{v+\sigma_{J} J, \sigma_{I} I}(A)^{-1} h_{v, \sigma_{J} J}(A)^{-1} h_{v, \sigma_{K} K}(A)\left[h_{v, \sigma_{K} K}(A)^{-1}, \hat{V}\right]\right)
\end{aligned}
$$

summed over all non-planar triples of edges in all possible orientations. (There are 48 terms in the sum, but we need to divide only by 8 since a factor of six arises in the contraction of basic fields occurring in the constraint.) The combination

$$
h_{v, \sigma_{I} I}(A) h_{v+\sigma_{I} I, \sigma_{J} J}(A) h_{v+\sigma_{J} J, \sigma_{I} I}(A)^{-1} h_{v, \sigma_{J} J}(A)^{-1}
$$

gives a single plaquette holonomy with tangent vectors $e_{v, \sigma_{I} I}$ and $e_{v, \sigma_{J} J}$ as illustrated in Fig. 2,

When expanded in $\ell_{0}$ assuming sufficiently small edges, the leading term is of the order $\ell_{0}^{3}$ which automatically results in a Riemann sum representation of the first term in (15). This justifies $\hat{H}^{(1)}$ as a quantization of the classical expression. As seen from the argument, one needs to assume that the lattice is sufficiently fine for classical values of the fields $A_{a}^{i}$. Thus, there are states corresponding to coarser lattices on which stronger quantum corrections can result. As usually, semiclassical behavior is not realized on all states but only for a select class. For any low-curvature classical configuration, one can make sure that a chosen lattice leads only to small quantum corrections such that sufficiently many semiclassical states exist. 


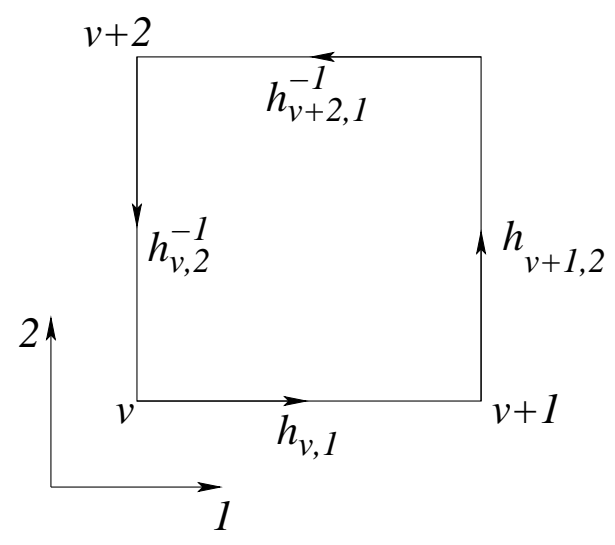

Figure 2: Elementary lattice plaquette with holonomies around a closed loop.

\subsubsection{Quantization}

The required calculations for $\mathrm{SU}(2)$ holonomies and their products usually do not allow explicit diagonalizations of operators. But some physical regimes allow one to decouple the matrix components at least approximately. This is realized for several symmetric models and also for perturbations at least of some metric modes around them. In particular, after splitting off the non-diagonal part of the connection in the perturbative expansion considered here, we can take the trace explicitly and reduce the expression to U(1). Since the diagonal part of the Ashtekar connection for our perturbations is contributed entirely by extrinsic curvature, we are effectively using "holonomies" computed for extrinsic curvature rather than the Ashtekar connection. Although extrinsic curvature is a tensor rather than a connection, it is meaningful to use it in expressions resembling holonomies, denoted here simply as $h_{v, I}$, on a given metric background. This has the additional advantage of easily combining the remaining quadratic terms in $K_{a}^{i}$ with the first term of the constraint (15) without using squares of multiple commutators from quantizing (16). Writing

$$
\begin{aligned}
F_{a b}^{i} & =2 \partial_{[a} \Gamma_{b]}^{i}+2 \gamma \partial_{[a} K_{b]}^{i}+\epsilon_{i j k}\left(\Gamma_{a}^{j}+\gamma K_{a}^{j}\right)\left(\Gamma_{b}^{k}+\gamma K_{b}^{k}\right) \\
& =2 \partial_{[a} \Gamma_{b]}^{i}+2 \gamma \partial_{[a} K_{b]}^{i}+\gamma \epsilon_{i j k}\left(\Gamma_{a}^{j} K_{b}^{k}+\Gamma_{b}^{k} K_{a}^{j}\right)+\epsilon_{i j k}\left(\Gamma_{a}^{j} \Gamma_{b}^{k}+\gamma^{2} K_{a}^{j} K_{b}^{k}\right)
\end{aligned}
$$

we obtain a term $2 \gamma \partial_{[a} K_{b]}^{i}+\gamma^{2} K_{a}^{j} K_{b}^{k}$ resembling "curvature" $F_{a b}^{i}(K)$ as computed from extrinsic curvature alone, a curvature term of the spin connection as well as cross-terms $\epsilon_{i j k}\left(\Gamma_{a}^{j} K_{b}^{k}+\Gamma_{b}^{k} K_{a}^{j}\right)$. In our context due to the diagonality conditions the cross-terms disappear [5] and we only have the $K$-curvature term and spin connection terms to quantize. The first term can then be combined with the term quadratic in $K_{a}^{i}$ in (15), removing the need to use double commutators. We denote this contribution to the constraint as

$$
H_{K}[N]:=\frac{1}{8 \pi G} \int_{\Sigma} \mathrm{d}^{3} x N|\operatorname{det} E|^{-1 / 2}\left(\epsilon_{i j k} \gamma \partial_{a} K_{b}^{i}-K_{a}^{j} K_{b}^{k}\right) E_{j}^{[a} E_{k}^{b]}
$$


(since also $\partial_{a} K_{b}^{i}$ drops out as used later, the constraint is $\gamma$-independent) and the remaining term as

$$
H_{\Gamma}[N]:=\frac{1}{8 \pi G} \int_{\Sigma} \mathrm{d}^{3} x N|\operatorname{det} E|^{-1 / 2}\left(\epsilon_{i j k} \partial_{a} \Gamma_{b}^{i}+\Gamma_{a}^{j} \Gamma_{b}^{k}\right) E_{j}^{[a} E_{k}^{b]} .
$$

Then, $H[N]=H_{K}[N]+H_{\Gamma}[N]$ is the constraint for scalar modes in longitudinal gauge. Both terms can rather easily be dealt with, using holonomies around a loop for the first term (this subsection) and direct quantizations of $\Gamma_{a}^{i}$ for the second (Sec. 3.1.4). The split-off spin connection components are thus quantized separately, which is possible in the perturbative treatment on a background, and then added on to the operator.

Note also that as a further simplification the derivative term of extrinsic curvature disappears from the constraint for diagonal variables as assumed here. This will automatically happen also from holonomies around loops. We emphasize that the quantization procedure followed is special to the given context of scalar perturbations on a flat isotropic background. Nevertheless, it mimics essential steps of the full constructions as discussed in more detail in Sec. 4.2.1. Its main advantage is that it allows explicit derivations of all necessary terms and thus explicit effective equations to be confronted with observations. Moreover, it is far from clear that the constructions currently done in the full setting will remain unchanged with further developments. We thus evaluate the key features of the scheme without paying too close attention to current details.

Following the general procedure, we thus obtain vertex contributions

$$
\begin{aligned}
\hat{H}_{K, v}= & -\frac{1}{16 \pi G} \frac{2 i}{8 \pi \gamma^{3} G \hbar} \frac{N(v)}{8} \sum_{I J K} \sum_{\sigma_{I} \in\{ \pm 1\}} \sigma_{1} \sigma_{2} \sigma_{3} \epsilon^{I J K} \\
& \times \operatorname{tr}\left(h_{v, \sigma_{I} I} h_{v+\sigma_{I} I, \sigma_{J} J} h_{v+\sigma_{J} J, \sigma_{I} I}^{-1} h_{v, \sigma_{J} J}^{-1} h_{v, \sigma_{K} K}\left[h_{v, \sigma_{K} K}^{-1}, \hat{V}_{v}\right]\right) .
\end{aligned}
$$

As before, $h_{v, I}$ denotes a $K$-holonomy along the edge oriented in the positive $I$-direction and starting at vertex $v$, but we also include the opposite direction $h_{v,-I}$ in the sum to ensure rotational invariance. Note that following our convention, such holonomies are identified with $h_{v-I, I}^{-1}$. In some of the holonomies, $v+I$ is again the vertex adjacent to $v$ in the positive $I$-direction. The $\{I J K\}$-summation is taken over all possible orientations of the $I J$-loop and a transversal $K$-direction. Also, for notational brevity, we introduce

$$
c_{v, I}:=\frac{1}{2} \operatorname{tr}\left(h_{v, I}\right) \quad, \quad s_{v, I}:=-\operatorname{tr}\left(\tau_{(I)} h_{v, I}\right)
$$

such that (5) becomes $h_{v, I}=c_{v, I}+2 \tau_{I} s_{v, I}$. In a continuum approximation, we have

$$
c_{v, I}=\cos \left(\gamma k_{I}(v+I / 2) / 2\right) \quad, \quad s_{v, I}=\sin \left(\gamma k_{I}(v+I / 2) / 2\right)
$$

where $k_{I}(v)=\ell_{0} \tilde{k}_{I}(v)$. After substituting this expression into (21) and making use of the identity 3 (for some fixed $I, J, K$ and numbers $x_{i}$ and $y_{i}$ )

$$
\begin{aligned}
\epsilon_{I J K} \operatorname{tr}\left[\left(x_{1} \mathbb{I}+2 y_{1} \tau_{I}\right)\left(x_{2} \mathbb{I}+2 y_{2} \tau_{J}\right)\left(x_{3} \mathbb{I}+2 y_{3} \tau_{K}\right)\right] & =\epsilon_{I J K} \operatorname{tr}\left(x_{1} x_{2} x_{3} \mathbb{I}\right)+8 \epsilon_{I J K} \operatorname{tr}\left(y_{1} y_{2} y_{3} \tau_{I} \tau_{J} \tau_{K}\right) \\
& =2\left(x_{1} x_{2} x_{3}-y_{1} y_{2} y_{3} \epsilon_{I J K}\right) \epsilon_{I J K},
\end{aligned}
$$

\footnotetext{
${ }^{3}$ Here the fundamental representation of $\tau_{I}$ has been used: $\operatorname{tr}(\mathbb{I})=2, \operatorname{tr}\left(\tau_{I} \tau_{J}\right)=-\frac{1}{2} \delta_{I J}$
} 
any one term of the sum in (21) becomes

$$
\begin{aligned}
& \frac{i}{8 \pi \gamma G \hbar} \operatorname{tr}\left(h_{v, I} h_{v+I, J} h_{v+J, I}^{-1} h_{v, J}^{-1} h_{v, K}\left[h_{v, K}^{-1}, \hat{V}_{v}\right]\right) \\
= & -\epsilon_{I J K}\left\{\left[\left(c_{v, I} c_{v+J, I}+s_{v, I} s_{v+J, I}\right) c_{v, J} c_{v+I, J}+\left(c_{v, I} c_{v+J, I}-s_{v, I} s_{v+J, I}\right) s_{v, J} s_{v+I, J}\right] \hat{A}_{v, K}\right\} \\
& +\epsilon_{I J K}^{2}\left\{\left[\left(c_{v, I} s_{v+J, I}-s_{v, I} c_{v+J, I}\right) s_{v, J} c_{v+I, J}+\left(s_{v, I} c_{v+J, I}+c_{v, I} s_{v+J, I}\right) c_{v, J} s_{v+I, J}\right] \hat{B}_{v, K}\right\},
\end{aligned}
$$

where

$$
\begin{aligned}
\hat{A}_{v, K} & :=\frac{1}{4 \pi i \gamma G \hbar}\left(\hat{V}_{v}-c_{v, K} \hat{V}_{v} c_{v, K}-s_{v, K} \hat{V}_{v} s_{v, K}\right), \\
\hat{B}_{v, K} & :=\frac{1}{4 \pi i \gamma G \hbar}\left(s_{v, K} \hat{V}_{v} c_{v, K}-c_{v, K} \hat{V}_{v} s_{v, K}\right) .
\end{aligned}
$$

In the first line of (24), the expression inside the curly braces is symmetric in the indices $I$ and $J$, hence vanishes when contracted with $\epsilon_{I J K}$. Therefore only the second line contributes, and the extrinsic curvature part of the gravitational constraint is

$$
\begin{aligned}
\hat{H}_{K, v}= & \frac{-N(v)}{64 \pi \gamma^{2} G} \sum_{I J K} \sum_{\sigma_{I} \in\{ \pm 1\}}\left\{\left[\left(c_{v, \sigma_{I} I} s_{v+\sigma_{J} J, \sigma_{I} I}-s_{v, \sigma_{I} I} c_{v+\sigma_{J} J, \sigma_{I} I}\right) s_{v, \sigma_{J} J} c_{v+\sigma_{I} I, \sigma_{J} J}\right.\right. \\
& \left.\left.+\left(s_{v, \sigma_{I} I} c_{v+\sigma_{J} J, \sigma_{I} I}+c_{v, \sigma_{I} I} s_{v+\sigma_{J} J, \sigma_{I} I}\right) c_{v, \sigma_{J} J} s_{v+\sigma_{I} I, \sigma_{J} J}\right] \hat{B}_{v, \sigma_{K} K}\right\} \\
= & \frac{-N(v)}{64 \pi \gamma^{2} G} \sum_{I J K} \sum_{\sigma_{I} \in\{ \pm 1\}}\left\{\left[s_{v, \sigma_{I} I, \sigma_{J} J}^{-} s_{v, \sigma_{J} J} c_{v+\sigma_{I} I, \sigma_{J} J}+s_{v, \sigma_{I} I, \sigma_{J} J}^{+} c_{v, \sigma_{J} J} s_{v+\sigma_{I} I, \sigma_{J} J}\right] \hat{B}_{v, \sigma_{K} K}\right\},
\end{aligned}
$$

where in the last line trigonometric identities have been used to express products of sines and cosines through

$$
s_{v, \sigma_{I} I, \sigma_{J} J}^{ \pm}:=\sin \left(\frac{\gamma}{2}\left(k_{\sigma_{I} I}\left(v+\sigma_{I} I / 2\right) \pm k_{\sigma_{I} I}\left(v+\sigma_{J} J+\sigma_{I} I / 2\right)\right) .\right.
$$

As in this expression, all functions $k_{I}$ are, as before, evaluated at the midpoint of the edge $e_{v, I}$. We see that in the homogeneous case the first term in the sum vanishes and the leading contribution is

$$
4 \sin \left(\gamma k_{I} / 2\right) \cos \left(\gamma k_{I} / 2\right) \sin \left(\gamma k_{J} / 2\right) \cos \left(\gamma k_{J} / 2\right) \hat{B}_{v, K}
$$

in agreement with [2].

\subsubsection{Higher curvature corrections}

There are two types of corrections visible from this expression: Using commutators to quantize inverse densitized triad components implies eigenvalues of $\hat{B}_{v, I}$ which differ from the classical expectation at small labels $\mu_{v, I}$. Moreover, using holonomies contributes higher order terms in extrinsic curvature together with higher spatial derivatives when sines and 
cosines are expanded in small curvature regimes. We will now compute the next-leading terms of higher powers and spatial derivatives of $\tilde{k}_{I}(v)$ before dealing with inverse power corrections in the following subsection.

First, recall the usual expectation that quantum gravity gives rise to low energy effective actions with higher curvature terms such as $\int \mathrm{d}^{4} x \sqrt{|\operatorname{det} g|} \ell_{\mathrm{P}}^{2} R^{2}$ or $\int \mathrm{d}^{4} x \sqrt{|\operatorname{det} g|} \ell_{\mathrm{P}}^{2} R_{\mu \nu \rho \sigma} R^{\mu \nu \rho \sigma}$ added to the Einstein-Hilbert action $\int \mathrm{d}^{4} x \sqrt{|\operatorname{det} g|} R$. Irrespective of details of numerical coefficients, there are two key aspects: The Planck length $\ell_{\mathrm{P}}=\sqrt{G \hbar}$ must be involved for dimensional reasons in the absence of any other length scale, and higher spatial as well as time derivatives arise with higher powers of $R_{\mu \nu \rho \sigma}$. In canonical variables, one expects higher powers and higher spatial derivatives of extrinsic curvature and the triad, together with components of the inverse metric necessary to define scalar quantities from higher curvature powers (which forces one to raise indices on the Riemann tensor, for instance). Higher time derivatives, on the other hand, are more difficult to see in a canonical treatment and correspond to the presence of independent quantum variables without classical analog [13].

Any quantization such as that followed here starts from the purely classical action where $\hbar$ and thus $\ell_{\mathrm{P}}$ vanishes. In effective equations of the resulting quantum theory, quantum corrections depending on $\hbar$ will nevertheless emerge. As a first step in deriving such effective equations, we have non-local holonomy terms in a Hamiltonian operator which through its expectation values in semiclassical states will give rise to similar contributions of the same functional form of $k_{I}(v)$. At first sight, however, the expressions above do not agree with expectations from higher curvature actions: One can easily see that in (26) there are higher powers of extrinsic curvature by expanding the trigonometric functions, and higher spatial derivatives of extrinsic curvature by Taylor expanding the discrete displacement involved, e.g., in $k_{I}(v+I / 2)$. Moreover, higher spatial derivatives of the triad arise from similar non-local terms in the spin connection contribution $\hat{H}_{\Gamma}$ discussed later. But there are no factors of the Planck length in such higher powers (all factors of $G$ and $\hbar$ are written out explicitly and not "set equal to one"). In fact, by definition $k_{I}(v)$ is dimensionless since it is obtained by multiplying the curvature component $\tilde{k}_{I}(v)$ with $\ell_{0}$ in which all possible dimensions cancel. Higher power terms here thus do not need any dimensionful prefactor. Moreover, there are no components of the inverse metric (which would be $1 / \tilde{p}^{I}(v)$ for our diagonal triads) in contrast to what is required in higher curvature terms.

Curvature expansion. To see how this is reconciled, we expand the Hamiltonian explicitly in $\ell_{0}$ after writing $k_{I}=\ell_{0} \tilde{k}_{I}$. This corresponds to a slowly varying field approximation with respect to the lattice size. For the $(+I,+J)$-plaquette, a single term in the sum (21) becomes

$$
\begin{array}{r}
2 s_{v, I, J}^{-} s_{v, J} c_{v+I, J}+2 s_{v, I, J}^{+} c_{v, J} s_{v+I, J}=\gamma^{2} \ell_{0}^{2} \tilde{k}_{I} \tilde{k}_{J}+\frac{1}{2} \gamma^{2} \ell_{0}^{3}\left(\tilde{k}_{I} \tilde{k}_{J, J}+\tilde{k}_{J} \tilde{k}_{I, I}+2 \tilde{k}_{J} \tilde{k}_{I, I}\right) \\
+\frac{1}{8} \gamma^{2} \ell_{0}^{4}\left(\tilde{k}_{I} \tilde{k}_{J, J J}+\tilde{k}_{J} \tilde{k}_{I, I I}+4\left(\tilde{k}_{I} \tilde{k}_{J, I I}+\tilde{k}_{I} \tilde{k}_{J, I J}+\tilde{k}_{I, I} \tilde{k}_{J, I}+\tilde{k}_{I, J} \tilde{k}_{J, I}\right)\right.
\end{array}
$$




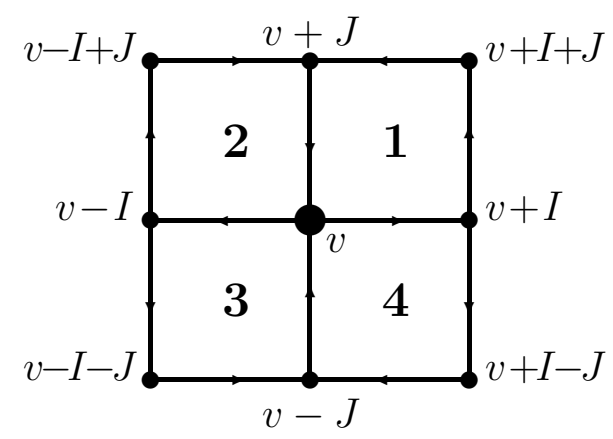

Figure 3: Four plaquettes adjacent to vertex $v$ in the $(I, J)$-plane. The arrows indicate the directions in which the relevant holonomies are traversed.

$$
\left.+2 \tilde{k}_{I, I} \tilde{k}_{J, J}-\frac{4}{3} \gamma^{2} \tilde{k}_{I} \tilde{k}_{J}\left(\tilde{k}_{I}^{2}+\tilde{k}_{J}^{2}\right)\right)+O\left(\ell_{0}^{5}\right) .
$$

(Commas on the classical field $\tilde{k}_{I}$ indicate partial derivatives along a direction given by the following index.) For a fixed direction $K$ there are in total eight terms to be included in the sum (21). They are obtained from (28) by taking into account the four plaquettes in the $(I, J)$-plane meeting at vertex $v$ (Fig. 3) and considering both orientations in which each plaquette can be traversed. While the latter merely boils down to symmetrization over $I$ and $J$, the former requires some care, noting that in the Hamiltonian constraint (21) $h_{v,-I}$ means $h_{v-I, I}^{-1}$. The contribution (28) corresponds to plaquette 1 of Fig. 3 and has $\sigma_{I}=\sigma_{J}=\sigma_{K}=1$. Accounting for the overall sign dictated by the $\sigma$-factors, one can obtain the expressions for the three remaining plaquettes 2, 3 and 4 following the recipe below.

\begin{tabular}{|c|c|c|c|c|c|}
\hline plaquette & \multicolumn{4}{|c|}{ extrinsic curvature components } & $\operatorname{sign}$ \\
\hline$(1)$ & $I / 2)$ & $k_{J}(v+I+J / 2)$ & $-k_{I}(v+I / 2+J)$ & $J / 2)$ & \\
\hline$(2)$ & $-k_{I}(v-I / 2)$ & $k_{J}(v-I+J / 2)$ & $k_{I}(v-I / 2+J)$ & $-k_{J}(v+J / 2)$ & $(-1)$ \\
\hline$(3)$ & $-k_{I}(v-I / 2)$ & $-k_{J}(v-I-J / 2)$ & $k_{I}(v-I / 2-J)$ & $k_{J}(v-J / 2)$ & $(-1)^{2}$ \\
\hline (4) & $k_{I}(v+I / 2)$ & $-k_{J}(v+I-J / 2)$ & $-k_{I}(v+I / 2-J)$ & $k_{J}(v-J / 2)$ & $(-1)$ \\
\hline
\end{tabular}

The first column designates a plaquette number, whereas the last one indicates the overall sign factor. The other four columns show the correspondence between the relevant link labels.

After the symmetrization over all four plaquettes (traversed in both directions), the cubic terms drop out

$$
\begin{aligned}
\gamma^{2} \ell_{0}^{2} \tilde{k}_{I} \tilde{k}_{J} & -\frac{\gamma^{4} \ell_{0}^{4}}{6} \tilde{k}_{I} \tilde{k}_{J}\left(\tilde{k}_{I}^{2}+\tilde{k}_{J}^{2}\right) \\
& +\frac{\gamma^{2} \ell_{0}^{4}}{8}\left(\tilde{k}_{I} \tilde{k}_{J, J J}+\tilde{k}_{J} \tilde{k}_{I, I I}+2\left(\tilde{k}_{I} \tilde{k}_{J, I I}+\tilde{k}_{J} \tilde{k}_{I, J J}+\tilde{k}_{I, I} \tilde{k}_{J, I}+\tilde{k}_{I, J} \tilde{k}_{J, J}\right)\right)+O\left(\ell_{0}^{5}\right) .
\end{aligned}
$$

Note that the link labels $\tilde{k}$ were introduced as values of the extrinsic curvature components evaluated at midpoints of edges in the continuum approximation (23) of our basic non-local 
variables. The expression above is written in terms of just two components $\tilde{k}_{I}(v)$ and $\tilde{k}_{J}(v)$ (and their partial spatial derivatives) Taylor expanded around the vertex $v$. The first term, when combined with $\hat{B}_{v, K}$ and summed over all triples $I J K$, reproduces the correct classical limit of the constraint $H_{K}$. This limit is obtained in two steps: we first performed the continuum approximation by replacing holonomies with mid-point evaluations of extrinsic curvature components. This would still give us a non-local Hamiltonian since each vertex contribution now refers to evaluations of the classical field at different points. In a second step we then Taylor expanded these evaluations around the central vertex $v$, which gives a local result and corresponds to a further, slowly-varying field approximation.

Comparison with higher curvature terms. Here, the factor $\ell_{0}^{2}$ in the leading term together with a factor $\ell_{0}$ from $\hat{B}_{v, K}$ through (14) combines to give the Riemann measure of the classical integral. Higher order terms, however, come with additional factors of $\ell_{0}$ in (29) which are not absorbed in this way. The result is certainly independent of coordinates since the whole construction (26) in terms of $k_{I}$ is coordinate independent. But for a comparison with higher curvature terms we have to formulate corrections in terms of $\tilde{k}_{I}$ and $\tilde{p}^{I}$ as these are the components of classical extrinsic curvature and densitized triad tensors. Higher order terms in the expansion are already formulated with $\tilde{k}_{I}$ in coordinate independent combinations with $\ell_{0}$-factors. It remains to interpret the additional $\ell_{0}$ factors appropriately for a comparison with low energy effective actions.

This can be done quite simply in a way which removes the above potential discrepancies between our expansions and higher curvature terms in low energy effective actions. We simply use (6) to write $\ell_{0}^{2}=p^{I} / \tilde{p}^{I}$ which is the only well-defined possibility to express $\ell_{0}$ in terms of the fields. Thus, inverse metric components $1 / \tilde{p}^{I}$ directly occur in combination with $\tilde{k}_{J}$ factors as required for higher curvature terms. The fact that the cubic term in $\ell_{0}$ in (29) drops out is also in agreement with higher curvature corrections since in that case only even powers of the length scale $\ell_{\mathrm{P}}$ occur. Moreover, there are now factors of $p^{I}$ multiplying the corrections. These are basic variables of the quantum theory determining the fundamental discreteness. Thus, factors of the Planck length occurring in low energy effective actions are replaced by the state specific quantities $p^{I}$. While the Planck length $\ell_{\mathrm{P}}=\sqrt{G \hbar}$ is expected to appear for dimensional reasons without bringing in information about quantum gravity (it can just be computed using classical gravity for $G$ and quantum mechanics for $\hbar$ ), the $p^{I}$ are determined by a state of quantum gravity. If expressed through labels $\mu_{v, I}$, the Planck length also appears, but it can be enlarged when $\mu_{v, I}>1$. Moreover, the lattice labels are dynamical (and in general inhomogeneous) and can thus change in time in contrast to $\ell_{\mathrm{P}}$. Although the form of corrections is analogous to those for low energy effective actions, the conceptual as well as dynamical appearance of correction terms is thus quite different.

The terms considered so far could not give rise to higher time derivatives of the spatial metric. In general, higher time derivatives describe the effect of quantum variables (3) of the field theory, which appear in the expectation value of the Hamiltonian constraint in a generic state. Quantum variables are thus, in a certain sense, analogous to higher time 
derivatives in effective actions [13], which indicates that the correction terms they imply should combine with those in (29) obtained by expanding sines and cosines to higher powers of space-time curvature components. All corrections of these types should thus be considered together since they will eventually be mixed up despite of their different derivations. A computation of terms containing quantum variables requires more detailed information about the expectation value of the constraint operator in an arbitrary state. These terms are thus more difficult to compute, which also makes an interpretation of the remaining higher curvature terms alone, especially concerning their possible covariance, more difficult 4 We will thus focus from now on on corrections coming from commutators $\hat{B}_{v, K}$ to quantize inverse powers which are independent of the higher order corrections and even give rise to non-perturbative terms. Moreover, in Sec. 5.2 .2 below we will demonstrate that those corrections are expected to be dominant in cosmological perturbation theory.

\subsubsection{Inverse triad corrections}

A direct calculation using (8) and (12) shows that $\hat{B}_{v, K}$ commutes with all flux operators and thus has flux eigenstates as eigenbasis, as it happens also in homogeneous models [4]. The action

$$
\begin{aligned}
\hat{B}_{v, K}\left|\ldots, \mu_{v, K}, \ldots\right\rangle & :=\left(2 \pi \gamma \ell_{\mathrm{P}}^{2}\right)^{1 / 2} \sqrt{\left|\mu_{v, I}+\mu_{v,-I}\right|\left|\mu_{v, I}+\mu_{v,-J}\right|} \\
& \times\left(\sqrt{\left|\mu_{v, K}+\mu_{v,-K}+1\right|}-\sqrt{\left|\mu_{v, K}+\mu_{v,-K}-1\right|}\right)\left|\ldots, \mu_{v, K}, \ldots\right\rangle
\end{aligned}
$$

directly shows the eigenvalues which do not agree exactly with the classical expectation $e_{K}(v)=\sqrt{\left|p^{I}(v) p^{J}(v) / p^{K}(v)\right|} \sim \sqrt{\left|\mu_{v, I} \mu_{v, J} / \mu_{v, K}\right|}$ (indices such that $\epsilon^{I J K}=1$ ) for the co-triad (13) which appears as a factor in the Hamiltonian constraint. But for large values $\mu_{v, I} \gg 1$ the classical expectation is approached as an expansion of the eigenvalues shows.

Inverse triad corrections are obtained by extracting the corrections which $B_{v, K}$ receives on smaller scales. We introduce the correction function as a factor $\alpha_{v, K}$, depending on the lattice labels $\mu_{v, I}$, such that $B_{v, K}=\alpha_{v, K} e_{v, K}$ and $\alpha_{v, K} \rightarrow 1$ classically, i.e. for $\mu_{v, K} \gg$ 1. Comparing the eigenvalues of $\hat{B}_{v, K}$ with those of flux operators in the combination $\sqrt{\left|\mathcal{F}_{v, I} \mathcal{F}_{v, J} / \mathcal{F}_{v, K}\right|}$, we find

$$
\alpha_{v, K}=\sqrt{\left|\mu_{v, K}+\mu_{v,-K}\right|}\left(\sqrt{\left|\mu_{v, K}+\mu_{v,-K}+1\right|}-\sqrt{\left|\mu_{v, K}+\mu_{v,-K}-1\right|}\right)
$$

\footnotetext{
${ }^{4}$ It is sometimes tempting to "sum the whole perturbations series" of higher order terms simply by using the left hand side of (28) directly in effective equations without an expansion. However, this is in general not a consistent approximation since arbitrarily small higher order terms are included but other types of corrections such as higher time derivatives or quantum variables are completely ignored. There is currently only one known model where the procedure is correct since all quantum variables have been shown to decouple from the expectation values [20. But this model, a free isotropic scalar in a certain factor ordering of the constraint operator, is very special. Under modifications such as a scalar potential quantum variables do not decouple and their motion implies further correction terms in effective equations not captured in the trigonometric functions arising from holonomies.
} 
After having computed the operators and their eigenvalues, we can specialize the correction function to perturbations of the scalar mode. We reduce the number of independent labels by imposing $\mu_{v, I}+\mu_{v,-I}=\mu_{v, J}+\mu_{v,-J}$ for arbitrary $I$ and $J$. This corresponds to a metric proportional to the identity $\delta_{a b}$ for a scalar perturbation. We then assign a new variable $p(v)=2 \pi \gamma \ell_{\mathrm{P}}^{2}\left(\mu_{v, I}+\mu_{v,-I}\right)$ to each vertex $v$, which is independent of the direction of the edge $I$ and describes the diagonal part of the triad. Quantum numbers in eigenvalues of the lattice operators can then be replaced by $p(v)$, and the resulting functions compared with the classical ones. The remaining subscript $v$ indicates that the physical quantities are vertex-dependent, i.e. inhomogeneous. Then the averaging over the plaquette orientations in the constraint becomes trivial and the total correction reads

$$
\alpha_{v}=2 \sqrt{\left|\mu_{v}\right|}\left(\sqrt{\left|\mu_{v}+1 / 2\right|}-\sqrt{\left|\mu_{v}-1 / 2\right|}\right)
$$

i.e.

$$
\alpha[p(v)]=\frac{\sqrt{|p(v)|}}{2 \pi \gamma \ell_{\mathrm{P}}^{2}}\left(\sqrt{\left|p(v)+2 \pi \gamma \ell_{\mathrm{P}}^{2}\right|}-\sqrt{\left|p(v)-2 \pi \gamma \ell_{\mathrm{P}}^{2}\right|}\right) .
$$

We will continue analyzing these correction functions in Sec. 3.3 after having discussed how such functions also enter the spin connection and matter terms.

\subsubsection{Spin connection}

So far, the holonomies we used only contributed the extrinsic curvature terms to the Hamiltonian but no spin connection terms at all. In the procedure followed here, we thus have to quantize $\Gamma_{a}^{i}[E]$ directly which is possible in the perturbative regime where line integrals of the spin connection have covariant meaning. This gives rise to one further correction function in the effective expression of the spin connection

$$
\Gamma_{I}^{i}=-\epsilon^{i j k} e_{j}^{b}\left(\partial_{[I} e_{b]}^{k}+\frac{1}{2} e_{k}^{c} e_{a}^{l} \partial_{[c} e_{b]}^{l}\right),
$$

as it also contains a co-triad (13). Since the triad and its inverse have a diagonal form

$$
e_{i}^{I} \equiv \frac{E_{i}^{I}}{\sqrt{|\operatorname{det} E|}}=e^{(I)} \delta_{i}^{I}, \quad e_{I}=e_{(I)} \delta_{I}^{i}
$$

with the components given by

$$
e^{I}=\frac{p^{I}}{\sqrt{|\operatorname{det} E|}}=\left(e_{I}\right)^{-1}, \quad \operatorname{det} E=p^{I} p^{J} p^{K},
$$

the spin-connection simplifies to

$$
\Gamma_{I}^{i}=\epsilon_{I}^{i c} e^{(c)} \partial_{c} e_{(I)}
$$


In terms of components of a densitized triad it reads

$$
\Gamma_{I}^{i}=\frac{1}{2} \epsilon_{I}^{i j} \frac{p^{(j)}}{p^{(I)}}\left(\sum_{J} \frac{\partial_{j} p^{J}}{p^{J}}-2 \frac{\partial_{j} p^{I}}{p^{I}}\right) .
$$

Since there are many alternative choices in performing the quantization of such an object, but not much guidance from a potential operator in the full theory, we first discuss general aspects one can expect for the quantization of the spin connection in a simple version. It includes corrections of inverse densitized triad components by correction functions in each term of (38). We thus mimic a quantization to the extent that expectation values of classical expressions containing inverse powers of $p$ acquire a correction factor

$$
\frac{1}{p^{I}(v)} \rightarrow \frac{\beta_{I}(v)}{p^{I}(v)}
$$

where the correction functions $\beta_{I}$ are kept different from the function $\alpha$ used before because the object to be quantized is different. There will also be corrections from the discretization $\Delta_{I}$ of partial derivatives $\ell_{0} \partial_{I}$, but we ignore them in what follows for the same reason which allowed us to ignore such effects from the loop holonomy quantizing $F_{a b}^{i}$. The effective analog of (38) is then of the form

$$
\left(\Gamma_{I}^{i}\right)_{\mathrm{eff}}=\frac{1}{2} \epsilon_{I}^{i j} \beta^{I} \frac{p^{j}}{p^{I}}\left(\sum_{J} \beta^{J} \frac{\partial_{j} p^{J}}{p^{J}}-2 \beta^{I} \frac{\partial_{j} p^{I}}{p^{I}}\right) .
$$

At this stage the triad components, corresponding to different orientations, can be put equal to each other in effective equations, $p^{I}=p^{J}=p^{K}=p$. This implies an analogous relation between the correction functions $\beta_{I}=\beta_{J}=\beta_{K}=\beta_{0}$. Comparing (40) with the ansatz $\Gamma_{I}^{i}=\frac{1}{2} \epsilon_{I}^{i j} \beta \frac{\partial_{j} p}{p}$, we conclude that also the spin connection receives a correction function $\beta=\beta_{0}^{2}$.

For a precise quantization we observe that we need terms of the form $\ell_{0}^{2} \Gamma_{a}^{i} \Gamma_{b}^{j}$ and $\ell_{0}^{2} \partial_{a} \Gamma_{b}^{i}$ in the constraint since one factor $\ell_{0}$ of the Riemann measure will be absorbed in the commutator $\hat{B}_{v, I}$. To quantize $\ell_{0} \Gamma_{a}^{i}$, we combine $\ell_{0}$ with the partial derivative $\partial_{I}$ in (37) to approximate a lattice difference operator $\Delta_{I}$ defined by $\left(\Delta_{I} f\right)_{v}=f_{v+I}-f_{v}$ for any lattice function $f$. A well-defined lattice operator thus results once a prescription for quantizing the inverse triad has been chosen. One can again make use of Poisson identities for the classical inverse which, however, allows more freedom than for the combination of triad components we saw in the Hamiltonian constraint. Such a freedom, corresponding to quantization ambiguities, will also be encountered when we consider matter Hamiltonians. For any choice we obtain a well-defined operator which would not be available without the perturbative treatment since the full spin connection is not a tensorial object.

An explicit example can most easily be derived by writing the spin connection integrated along a link $e_{v, I}$ as it might appear in a holonomy,

$$
\int_{e_{v, I}} \dot{e}_{I}^{a} \Gamma_{a}^{i} \approx \ell_{0} \Gamma_{I}^{i}=\epsilon_{I}^{i c} e^{(c)} \ell_{0} \partial_{c} e_{(I)} \approx \epsilon_{I}^{i K} \frac{p^{(K)}}{\sqrt{|\operatorname{det} E|}} \Delta_{K} e_{(I)}
$$


using the lattice difference operator $\Delta_{I} \approx \ell_{0} \partial_{I}$. We then have to deal with the inverse powers explicit in the fraction and implicit in the co-triad $e_{I}$. The latter is standard, replacing $e_{I}$ by $\ell_{0}^{-1} h_{I}\left\{h_{I}^{-1}, V_{v}\right\}$ based on (13). The inverse determinant in the fraction cannot be absorbed in the resulting Poisson bracket because (i) it does not commute with the derivative and (ii) absorbing a single inverse in a single co-triad would lead to a logarithm of $V_{v}$ in the Poisson bracket which would not be well-defined. It can, however, be absorbed in the flux $\ell_{0}^{2} p^{K}$ if we do not use the basic flux operator $\hat{F}_{v, K}$ but the classically equivalent expression

$$
\begin{aligned}
F_{v, K} & \approx \ell_{0}^{2} p^{K}=\frac{1}{2} \ell_{0}^{2} \delta_{(K)}^{k} \epsilon_{k i j} \epsilon^{K I J} e_{I}^{i} e_{J}^{j} \\
& =-\frac{1}{4}(4 \pi \gamma G)^{-2} \sum_{I J} \sum_{\sigma_{I} \in\{ \pm 1\}} \sigma_{I} \sigma_{J} \epsilon^{I J K} \operatorname{tr}\left(\tau_{(K)} h_{I}\left\{h_{I}^{-1}, V_{v}\right\} h_{J}\left\{h_{J}^{-1}, V_{v}\right\}\right)
\end{aligned}
$$

which is analogous to expressions used in [21. Since there are two Poisson brackets, we can split the inverse $V_{v}$ evenly among them, giving rise to square roots of $V_{v}$ in the brackets:

$$
\begin{aligned}
\frac{p^{K}}{\sqrt{|\operatorname{det} E|}} & \approx \ell_{0} \frac{F_{v, K}}{V_{v}} \\
& =-\frac{\ell_{0}}{16 \pi^{2} \gamma^{2} G^{2}} \sum_{I J} \sum_{\sigma_{I} \in\{ \pm 1\}} \sigma_{I} \sigma_{J} \epsilon^{I J K} \operatorname{tr}\left(\tau_{(K)} h_{I}\left\{h_{I}^{-1}, \sqrt{V_{v}}\right\} h_{J}\left\{h_{J}^{-1}, \sqrt{V_{v}}\right\}\right)(
\end{aligned}
$$

The remaining factor of $\ell_{0}$ is absorbed in $e_{I}$ inside the derivative which is quantized following the standard procedure. A well-defined quantization of spin connection components thus follows, which is not local in a vertex since the difference operator connects to the next vertex. Similarly, the derivative of the spin connection needed in the Hamiltonian constraint leads to further connections to next-to-next neighbors.

Explicitly, one can thus write an integrated spin connection operator quantizing $\Gamma_{v, I}^{i}:=$ $\int_{e_{v, I}} \mathrm{~d} t \dot{e}_{I}^{a} \Gamma_{a}^{i}$ as

$$
\begin{aligned}
\hat{\Gamma}_{v, I}^{i}= & \epsilon_{I}^{i K}\left(\frac{1}{16 \pi^{2} \gamma^{2} \ell_{\mathrm{P}}^{2}} \sum_{J, L, \sigma_{J}, \sigma_{L}} \sigma_{J} \sigma_{L} \epsilon^{J L K} \operatorname{tr}\left(\tau_{(K)} h_{J}\left[h_{J}^{-1}, \hat{V}_{v}^{1 / 2}\right] h_{L}\left[h_{L}^{-1}, \hat{V}_{v}^{1 / 2}\right]\right)\right. \\
& \left.\times \Delta_{K}\left(\frac{i}{2 \pi \gamma \ell_{\mathrm{P}}^{2}} \operatorname{tr}\left(\tau^{(I)} h_{I}\left[h_{I}^{-1}, \hat{V}_{v}\right]\right)\right)\right) .
\end{aligned}
$$

Replacing the commutators by classical expressions times correction functions $\alpha$ defined as before and $\alpha_{1 / 2}$ defined similarly for a commutator containing the square root of the volume operator leads to an expression

$$
\begin{aligned}
\left(\Gamma_{I}^{i}\right)_{\mathrm{eff}} & =\alpha_{1 / 2}\left(p^{i}\right) \alpha_{1 / 2}\left(p^{I}\right) \epsilon_{I}^{i c} e^{(c)} \partial_{c}\left(\alpha\left(p^{I}\right) e_{I}\right) \\
& =\alpha_{1 / 2}\left(p^{i}\right) \alpha_{1 / 2}\left(p^{I}\right) \alpha\left(p^{I}\right) \Gamma_{I}^{i}+\alpha_{1 / 2}\left(p^{i}\right) \alpha_{1 / 2}\left(p^{I}\right) \alpha^{\prime}\left(p^{I}\right) e_{I} \epsilon_{I}^{i c} e^{(c)} \partial_{c} p^{(I)}
\end{aligned}
$$


where the prime denotes a derivative by $p^{I}$. Using the relation $p^{J}=e_{I} e_{K}$ whenever $\epsilon_{J I K}=1$ between densitized triad and co-triad components allows us to write

$$
\begin{aligned}
\left(\Gamma_{I}^{i}\right)_{\mathrm{eff}} & =\alpha_{1 / 2}\left(p^{i}\right) \alpha_{1 / 2}\left(p^{I}\right) \alpha\left(p^{I}\right) \Gamma_{I}^{i}+\left.\alpha_{1 / 2}\left(p^{i}\right) \alpha_{1 / 2}\left(p^{I}\right) \alpha^{\prime}\left(p^{I}\right) e_{I} \epsilon_{I}^{i c} e^{(c)} \partial_{c}\left(e_{J} e_{K}\right)\right|_{\epsilon_{I J K}=1} \\
& =\alpha_{1 / 2}\left(p^{i}\right) \alpha_{1 / 2}\left(p^{I}\right)\left(\alpha\left(p^{I}\right) \Gamma_{I}^{i}+\sum_{K \neq I} \alpha^{\prime}\left(p^{I}\right) p^{K} \Gamma_{K}^{i}\right)
\end{aligned}
$$

for the effective spin connection components. For scalar modes, using that all $p^{I}$ at a given point are equal, this can be written with a single correction function

$$
\beta[p(v)]=\alpha_{1 / 2}[p(v)]^{2}\left(\alpha[p(v)]+2 p \alpha^{\prime}[p(v)]\right)
$$

for $\Gamma_{I}^{i}$, where $\alpha^{\prime}=\mathrm{d} \alpha / \mathrm{d} p$.

\subsection{Matter Hamiltonian}

Matter fields are quantized by similar means in a loop quantization, using lattice states, and then coupled dynamically to geometry by adding the matter Hamiltonian to the constraint.

For a scalar field $\phi$, the momentum $\pi=\sqrt{|\operatorname{det} E|} \dot{\phi} / N$ is a density of weight one. In the $\phi$-representation, states will simply be of the form already used for the gravitational field, except that each vertex now also carries a label $\nu_{v} \in \mathbb{R}$ describing the dependence on the scalar field $\phi(v)$ through $\exp \left(i \nu_{v} \phi(v)\right)$ [22]. Well-defined lattice operators are then given by $\left.\widehat{\exp \left(i \nu_{0}\right.} \phi_{v}\right)$, for any $\nu_{0} \in \mathbb{R}$, which shifts the label $\nu_{v}$ by $\nu_{0}$. The momentum, with its density weight, has to be integrated before it can meaningfully be quantized. We introduce

$$
P_{v}:=\int_{R_{v}} \mathrm{~d}^{3} x \pi \approx \ell_{0}^{3} \pi(v)
$$

where $R_{v}$ is a cubic region around the vertex $v$ of the size of a single lattice site. Since we have $\left\{\phi(v), P_{w}\right\}=\chi_{R_{w}}(v)$ in terms of the characteristic function $\chi_{R}(v)=1$ if $v \in R$ and zero otherwise, a momentum operator $P_{v}$ must have eigenvalue $\hbar \nu_{v}$ in a state introduced above.

\subsubsection{Inverse triad corrections}

For the matter Hamiltonian of a scalar field $\phi$ with momentum $\pi$ and potential $U(\phi)$ we have the classical expression

$$
H_{\phi}[N]=\int \mathrm{d}^{3} x N(x)\left[\frac{1}{2 \sqrt{\operatorname{det}(q)}} \pi(x)^{2}+\frac{E_{i}^{a} E_{i}^{b}}{2 \sqrt{\operatorname{det} q}} \partial_{a} \phi(x) \partial_{b} \phi(x)+\sqrt{\operatorname{det} q} U(\phi)\right]
$$

containing inverse powers of the metric, too. It can be quantized by loop techniques [23, 24] making use of identities similar to (13). One first generalizes the identity to arbitrary positive powers of the volume in a Poisson bracket,

$$
\left\{A_{a}^{i}, V_{v}^{r}\right\}=4 \pi \gamma G r V_{v}^{r-1} e_{a}^{i}
$$


and then combines such factors with suitable exponents $r$ to produce a given product of triad and co-triad components. Since such identities would be used only when inverse components of densitized triads are involved and a positive power of volume must result in the Poisson bracket, the allowed range for $r$ is $0<r<2$. Any such Poisson bracket will be quantized to

$$
\dot{e}_{K}^{a}\left\{A_{a}^{i}, V_{v}^{r}\right\} \mapsto \frac{-2}{i \hbar \ell_{0}} \operatorname{tr}\left(\tau^{i} h_{v, K}\left[h_{v, K}^{-1}, \hat{V}_{v}^{r}\right]\right)
$$

using holonomies $h_{v, I}$ in direction $I$ with tangent vector $\dot{e}_{K}^{a}$. Since holonomies in our lattice states have internal directions $\tau_{K}$ for direction $K$, we can compute the trace and obtain

$$
\widehat{V_{v}^{r-1} e_{K}^{i}}=\frac{-2}{8 \pi i r \gamma \ell_{\mathrm{P}}^{2} \ell_{0}} \sum_{\sigma \in\{ \pm 1\}} \sigma \operatorname{tr}\left(\tau^{i} h_{v, \sigma K}\left[h_{v, \sigma K}^{-1}, \hat{V}_{v}^{r}\right]\right)=\frac{1}{2 \ell_{0}}\left(\hat{B}_{v, K}^{(r)}-\hat{B}_{v,-K}^{(r)}\right) \delta_{(K)}^{i}
$$

where, for symmetry, we use both edges touching the vertex $v$ along direction $K$ and $\hat{B}_{v, K}^{(r)}$ is the generalized version of (25):

$$
\hat{B}_{v, K}^{(r)}:=\frac{1}{4 \pi i \gamma G \hbar r}\left(s_{v, K} \hat{V}_{v}^{r} c_{v, K}-c_{v, K} \hat{V}_{v}^{r} s_{v, K}\right)
$$

The exponent used for the gravitational part was $r=1$, and $r=1 / 2$ already occurred in the spin connection, while the scalar Hamiltonians introduced in [23, 24], which we closely follow in the construction of the matter Hamiltonian here, use $r=1 / 2$ for the kinetic term and $r=3 / 4$ for the gradient term. With

$$
\epsilon^{a b c} \epsilon_{i j k}\left\{A_{a}^{i}, V_{v}^{1 / 2}\right\}\left\{A_{b}^{j}, V_{v}^{1 / 2}\right\}\left\{A_{c}^{k}, V_{v}^{1 / 2}\right\}=(2 \pi \gamma G)^{3} \epsilon^{a b c} \epsilon_{i j k} \frac{e_{a}^{i} e_{b}^{j} e_{c}^{k}}{V_{v}^{3 / 2}}=\frac{6(2 \pi \gamma G)^{3}}{\ell_{0}^{3} V_{v}^{1 / 2}}
$$

for a lattice site volume $V_{v} \approx \ell_{0}^{3}\left|\operatorname{det}\left(e_{a}^{i}\right)\right|$ and

$$
\epsilon^{a b c} \epsilon_{i j k}\left\{A_{b}^{j}, V_{v}^{3 / 4}\right\}\left\{A_{c}^{k}, V_{v}^{3 / 4}\right\}=(3 \pi \gamma G)^{2} \epsilon^{a b c} \epsilon_{i j k} \frac{e_{b}^{j} e_{c}^{k}}{V_{v}^{1 / 2}}=6(3 \pi \gamma G)^{2} \frac{E_{i}^{a}}{V_{v}^{1 / 2}}
$$

one can replace the inverse powers in the scalar Hamiltonian as follows: For the kinetic term, we discretize

$$
\int \mathrm{d}^{3} x \frac{\pi^{2}}{\sqrt{\operatorname{det} q}} \approx \sum_{v} \ell_{0}^{3} \frac{\pi(v)^{2}}{\sqrt{\operatorname{det} q(v)}} \approx \sum_{v} \frac{P_{v}^{2}}{V_{v}}
$$

Then, the classically singular

$$
\frac{1}{V_{v}}=\left(\frac{\ell_{0}^{3}}{6} \epsilon^{a b c} \epsilon_{i j k} \frac{e_{a}^{i} e_{b}^{j} e_{c}^{k}}{V_{v}^{3 / 2}}\right)^{2}=\left(\frac{\ell_{0}^{3}}{6(2 \pi \gamma G)^{3}} \epsilon^{a b c} \epsilon_{i j k}\left\{A_{a}^{i}, V_{v}^{1 / 2}\right\}\left\{A_{b}^{j}, V_{v}^{1 / 2}\right\}\left\{A_{c}^{k}, V_{v}^{1 / 2}\right\}\right)^{2}
$$

will be quantized to

$$
\left(\frac{1}{48} \epsilon^{I J K} \epsilon_{i j k}\left(\hat{B}_{v, I}^{(1 / 2)}-\hat{B}_{v,-I}^{(1 / 2)}\right) \delta_{(I)}^{i}\left(\hat{B}_{v, J}^{(1 / 2)}-\hat{B}_{v,-J}^{(1 / 2)}\right) \delta_{(J)}^{j}\left(\hat{B}_{v, K}^{(1 / 2)}-\hat{B}_{v,-K}^{(1 / 2)}\right) \delta_{(K)}^{k}\right)^{2}
$$


Similarly, we discretize the gradient term by

$$
\int \mathrm{d}^{3} x \frac{E_{i}^{a} E_{i}^{b}}{\sqrt{\operatorname{det} q}} \partial_{a} \phi \partial_{b} \phi \approx \sum_{v} \ell_{0}^{3} \frac{E_{i}^{a}(v) E_{i}^{b}(v)}{\sqrt{\operatorname{det} q(v)}}\left(\partial_{a} \phi\right)(v)\left(\partial_{b} \phi\right)(v) \approx \sum_{v} \frac{p^{I}(v) p^{J}(v)}{V_{v}} \Delta_{I} \phi_{v} \Delta_{J} \phi_{v}
$$

where we replace spatial derivatives $\partial_{a}$ by lattice differences $\Delta_{I}$. Now, using

$$
\delta_{(I)}^{i} \frac{p^{I}(v)}{V_{v}^{1 / 2}}=\ell_{0}^{2} \frac{E_{i}^{I}(v)}{V_{v}^{1 / 2}}=\frac{\ell_{0}^{2}}{6} \frac{\epsilon^{I b c} \epsilon_{i j k} e_{b}^{j} e_{c}^{k}}{V_{v}^{1 / 2}}=\frac{\ell_{0}^{2}}{6(3 \pi \gamma G)^{2}} \epsilon^{I b c} \epsilon_{i j k}\left\{A_{b}^{j}, V_{v}^{3 / 4}\right\}\left\{A_{c}^{k}, V_{v}^{3 / 4}\right\}
$$

we can quantize the metric contributions to the gradient term by

$$
\begin{aligned}
\frac{1}{24^{2}} & \epsilon^{I K L} \epsilon_{i j k}\left(\hat{B}_{v, K}^{(3 / 4)}-\hat{B}_{v,-K}^{(3 / 4)}\right) \delta_{(K)}^{j}\left(\hat{B}_{v, L}^{(3 / 4)}-\hat{B}_{v,-L}^{(3 / 4)}\right) \delta_{(L)}^{k} \\
& \times \epsilon^{J M N} \epsilon_{i m n}\left(\hat{B}_{v, M}^{(3 / 4)}-\hat{B}_{v,-M}^{(3 / 4)}\right) \delta_{(M)}^{m}\left(\hat{B}_{v, N}^{(3 / 4)}-\hat{B}_{v,-N}^{(3 / 4)}\right) \delta_{(N)}^{n} .
\end{aligned}
$$

In addition to the fact that we are using different values for $r$ in each term in the gravitational and matter parts, giving rise to different correction functions, the matter terms are less unique than the gravitational term and can be written with different parameters $r$. This corresponds to quantization ambiguities which will appear also in effective equations and which could have phenomenological implications. Some choices are preferred since they give rise to simpler expressions, but this does not suffice to determine a unique quantization. Instead of using $r=1 / 2$ in the kinetic term, for instance, we can use the class of relations

$$
\begin{gathered}
\frac{1}{\sqrt{|\operatorname{det} E|}}=\frac{(\operatorname{det} e)^{k}}{(\operatorname{det} E)^{(k+1) / 2}}=\left(\frac{1}{6} \epsilon^{a b c} \epsilon_{i j k}(4 \pi G \gamma)^{3}\right. \\
\left.\times\left\{A_{a}^{i}, V^{(2 k-1) / 3 k}\right\}\left\{A_{b}^{j}, V^{(2 k-1) / 3 k}\right\}\left\{A_{c}^{j}, V^{(2 k-1) / 3 k}\right\}\right)^{k}
\end{gathered}
$$

for any positive integer $k$ to write the inverse determinant through Poisson brackets not involving the inverse volume (see also the appendix of [25]). This determines an integer family of quantizations with $r_{k}=(2 k-1) / 3 k>\frac{1}{3}$. For $k=2$ we obtain the previous expression, but other choices are possible. Moreover, using the same $r$ in all terms arising in gravitational and matter Hamiltonians can only be done in highly contrived ways, if at all. There is thus no clearly distinguished value. From now on we will work with the choices specified above.

On regular lattice states, all ingredients are composed to a Hamiltonian operator

$$
\begin{aligned}
\hat{H}_{\phi}[N] & =\sum_{v \in \gamma} N_{v}\left[\frac{1}{2} \hat{P}_{v}^{2}\left(\frac{1}{48} \sum_{I J K, \sigma_{I} \in\{ \pm 1\}} \sigma_{1} \sigma_{2} \sigma_{3} \epsilon^{I J K} \hat{B}_{v, \sigma_{1} I}^{(1 / 2)} \hat{B}_{v, \sigma_{2} J}^{(1 / 2)} \hat{B}_{v, \sigma_{3} K}^{(1 / 2)}\right)^{2}\right. \\
& \left.+\frac{1}{2}\left(\frac{1}{48} \sum_{I J K, \sigma_{I} \in\{ \pm 1\}} \sigma_{1} \sigma_{2} \sigma_{3} \epsilon^{I J K}\left(\sigma_{1} \Delta_{\sigma_{1} I} \phi\right)_{v} \hat{B}_{v, \sigma_{2} J}^{(3 / 4)} \hat{B}_{v, \sigma_{3} K}^{(3 / 4)}\right)^{2}+\hat{V}_{v} U\left(\phi_{v}\right)\right]
\end{aligned}
$$




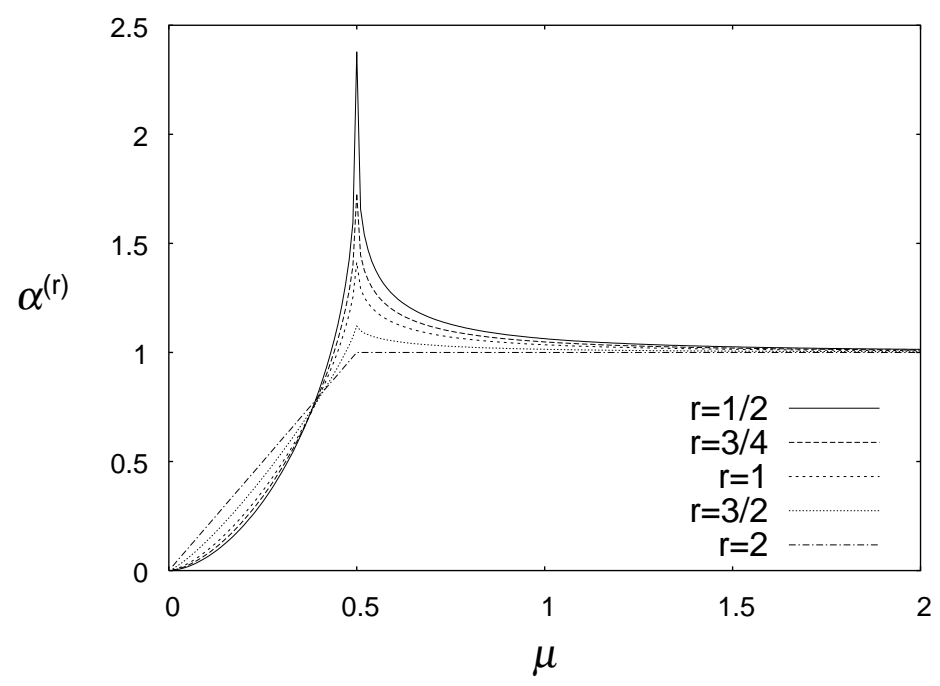

Figure 4: Behavior of the correction function $\alpha$. It approaches one from above for large arguments. For small arguments, the function is increasing from zero and reaches a peak value larger than one. Also shown is the limiting case $r=2$ which does not show a peak but a constant correction function for $\mu>1$.

\subsubsection{Matter correction functions}

As before, we compute eigenvalues of the operators

$$
\hat{B}_{v, K}^{(r)}:=\left(2 \pi \gamma \ell_{\mathrm{P}}^{2}\right)^{-1} \frac{\left.\hat{V}^{r}\right|_{\mu_{v, K}+1}-\left.\hat{V}^{r}\right|_{\mu_{v, K}-1}}{r}
$$

where the subscript of the volume operator indicates that its eigenvalue in a lattice state is computed according to (12) with a shifted label of link $e_{v, I}$. Thus, the eigenvalues are

$B_{v, K}^{(r)}:=\frac{1}{r}\left(2 \pi \gamma \ell_{\mathrm{P}}^{2}\right)^{\frac{3 r}{2}-1}\left|\mu_{v, I}+\mu_{v,-I}\right|^{r / 2}\left|\mu_{v, J}+\mu_{v,-J}\right|^{r / 2}\left(\left|\mu_{v, K}+\mu_{v,-K}+1\right|^{r / 2}-\left|\mu_{v, K}+\mu_{v,-K}-1\right|^{r / 2}\right)$.

compared to the classical expectation

$$
\left(2 \pi \gamma \ell_{\mathrm{P}}^{2}\right)^{\frac{3 r}{2}-1}\left|\mu_{v, I}+\mu_{v,-I}\right|^{r / 2}\left|\mu_{v, J}+\mu_{v,-J}\right|^{r / 2}\left|\mu_{v, K}+\mu_{v,-K}\right|^{r / 2-1}
$$

for $V^{r-1} e_{K}$. For any $r$, correction functions

$$
\alpha_{v, K}^{(r)}=\frac{1}{r}\left|\mu_{v, K}+\mu_{v,-K}\right|^{1-r / 2}\left(\left|\mu_{v, K}+\mu_{v,-K}+1\right|^{r / 2}-\left|\mu_{v, K}+\mu_{v,-K}-1\right|^{r / 2}\right)
$$

result. The main examples of $r$ are seen in Fig. 4.

Imposing again that $\mu_{v, I}+\mu_{v,-I}=\mu_{v, J}+\mu_{v,-J}$ for arbitrary $I$ and $J$ and introducing $p(v)=2 \pi \gamma \ell_{\mathrm{P}}^{2}\left(\mu_{v, I}+\mu_{v,-I}\right)$, we obtain the effective correction functions

$$
\alpha^{(r)}[p(v)]=\frac{2}{2 \pi r \gamma \ell_{\mathrm{P}}^{2}}|p(v)|^{1-r / 2}\left(\left|p(v)+2 \pi \gamma \ell_{\mathrm{P}}^{2}\right|^{r / 2}-\left|p(v)-2 \pi \gamma \ell_{\mathrm{P}}^{2}\right|^{r / 2}\right) .
$$


This can be used to write the effective matter Hamiltonian on a conformally flat space $q_{a b}=|p(x)| \delta_{a b}$ as

$$
H_{\phi}=\int_{\Sigma} \mathrm{d}^{3} x N(x)\left(\frac{D[p(x)]}{2|\tilde{p}(x)|^{3 / 2}} \pi(x)^{2}+\frac{\sigma[p(x)])|\tilde{p}(x)|^{\frac{1}{2}} \delta^{a b}}{2} \partial_{a} \phi \partial_{b} \phi+|\tilde{p}(x)|^{\frac{3}{2}} U(\phi)\right),
$$

where comparison with (51) shows that

$$
D[p(v)]=\alpha^{(1 / 2)}[p(v)]^{6} \quad \text { and } \quad \sigma[p(v)]=\alpha^{(3 / 4)}[p(v)]^{4} .
$$

\subsection{Properties of correction functions from inverse powers}

We have derived several different correction functions, making use of different parameters $r$. In most cases one could make different choices of such parameters and still write the classically intended expression in an equivalent way. This gives rise to quantization ambiguities since the eigenvalues of $\hat{B}_{v, K}^{(r)}$ depend on the value $r$, and so will correction functions. In addition to the ambiguities in the exponents $r$, one could use different representations for holonomies before taking the trace rather than only the fundamental representation understood above [26, 27]. In this case, we have more generally

$$
\hat{B}_{v, K}^{(r, j)}=\frac{3}{i r j(j+1)(2 j+1)}\left(2 \pi \gamma \ell_{\mathrm{P}}^{2}\right)^{-1} \operatorname{tr}_{j}\left(\tau^{K} h_{v, K} \hat{V}_{v}^{r} h_{v, K}^{-1}\right) .
$$

Eigenvalues of such operators can be expressed as

$B_{v, K}^{(r, j)}=\frac{3}{r j(j+1)(2 j+1)}\left(2 \pi \gamma \ell_{\mathrm{P}}^{2}\right)^{\frac{3 r}{2}-1}\left|\mu_{v, I}+\mu_{v,-I}\right|^{r / 2}\left|\mu_{v, J}+\mu_{v,-J}\right|^{r / 2} \sum_{m=-j}^{j} m\left|\mu_{v, K}+\mu_{v,-K}+2 m\right|^{r / 2}$

which leads to the general class of correction functions

$$
\alpha_{v, K}^{(r, j)}=\frac{3}{r j(j+1)(2 j+1)}\left|\mu_{v, K}+\mu_{v,-K}\right|^{1-\frac{r}{2}} \sum_{m=-j}^{j} m\left|\mu_{v, K}+\mu_{v,-K}+2 m\right|^{r / 2} .
$$

After imposing isotropy the last expression becomes

$$
\alpha^{(r, j)}=\frac{6}{r j(j+1)(2 j+1)}|\mu|^{1-\frac{r}{2}} \sum_{m=-j}^{j} m|\mu+m|^{r / 2}
$$

which is shown for a few cases in Fig. 5

For large $j$, the sum in $\alpha^{(r, j)}$ can be approximated using calculations as in [27]. The idea is to consider two cases: i) $\mu>j$ and ii) $\mu<j$ separately. In the former, the absolute values 


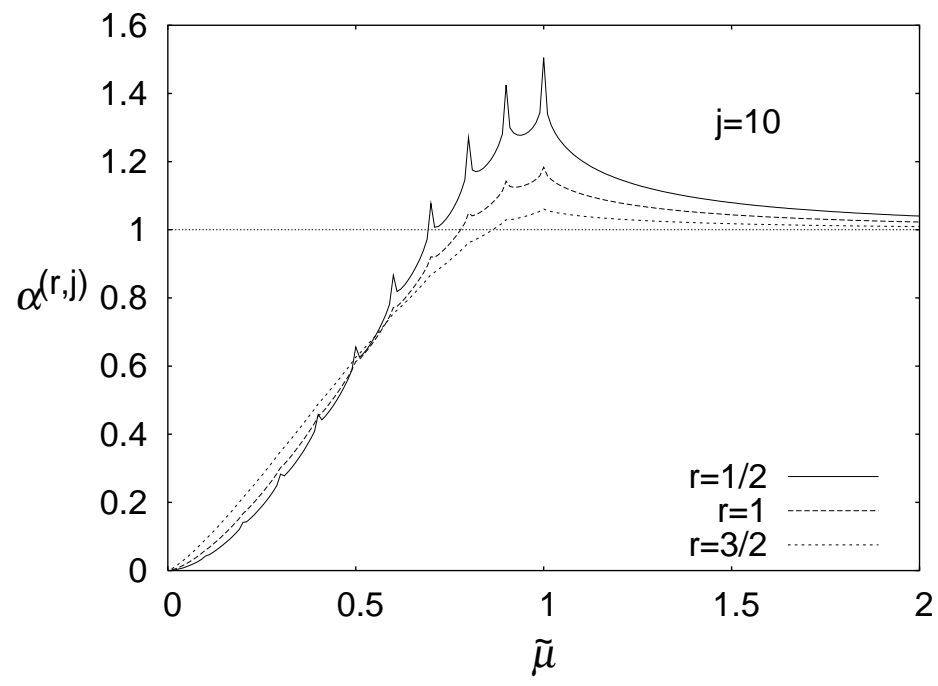

Figure 5: Behavior of the correction function $\alpha$ for larger $j$. The general trend is similar to the case for $j=1 / 2$, but there are $[j]+1$ spikes at $\mu=1, \ldots, j$ for integer $j$ and $\mu=1 / 2, \ldots, j$ otherwise. Above the peak, the function is smooth.

can be omitted as all the expressions under the sum are positive. Then the summation is to be replaced by integration to yield

$$
\begin{aligned}
\alpha^{(r, j)}=\frac{12 j^{3} \tilde{\mu}^{1-\frac{r}{2}}}{r j(j+1)(2 j+1)} & {\left[\frac{1}{r+4}\left((\tilde{\mu}+1)^{\frac{r}{2}+2}-(\tilde{\mu}-1)^{\frac{r}{2}+2}\right)\right.} \\
& \left.-\frac{\tilde{\mu}}{r+2}\left((\tilde{\mu}+1)^{\frac{r}{2}+1}-(\tilde{\mu}-1)^{\frac{r}{2}+1}\right)\right], \quad \tilde{\mu}>1
\end{aligned}
$$

where $\tilde{\mu}:=\mu / j$. In the second case, the terms in the sum corresponding to $m<\mu$ and $m>\mu$ should again be considered separately. The end result, however, is very similar to the previous one

$$
\begin{aligned}
& \alpha^{(r, j)}=\frac{12 j^{3} \tilde{\mu}^{1-\frac{r}{2}}}{r j(j+1)(2 j+1)}\left[\frac{1}{r+4}\left((\tilde{\mu}+1)^{\frac{r}{2}+2}-(1-\tilde{\mu})^{\frac{r}{2}+2}\right)\right. \\
& \left.-\frac{\tilde{\mu}}{r+2}\left((\tilde{\mu}+1)^{\frac{r}{2}+1}+(1-\tilde{\mu})^{\frac{r}{2}+1}\right)\right], \quad \tilde{\mu}<1
\end{aligned}
$$

After some rearrangements and using that $j \gg 1$ these two expressions can be combined into a single one as

$$
\alpha^{(r, j)}=\frac{6 \tilde{\mu}^{1-\frac{r}{2}}}{r(r+2)(r+4)}\left[(\tilde{\mu}+1)^{\frac{r}{2}+1}(r+2-2 \tilde{\mu})+\operatorname{sgn}(\tilde{\mu}-1)|\tilde{\mu}-1|^{\frac{r}{2}+1}(r+2+2 \tilde{\mu})\right] .
$$

The approximation is compared to the exact expression of the correction function obtained through eigenvalues in Fig. 6. As one can see, the spikes are smeared out by 


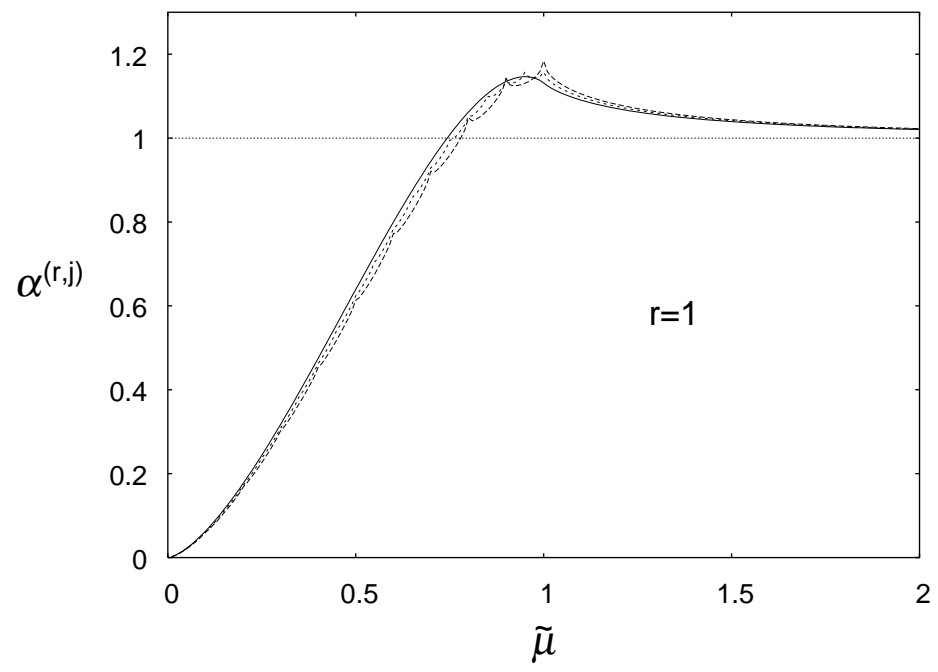

Figure 6: Comparison between the correction function (57) and its approximation (59). The spikes are smeared out by the approximation.

the approximation (except for the point $\tilde{\mu}=1$ where the approximation remains nondifferentiable at second order which is not visible from the plot). The general trend, however, is reproduced well even below the peak. For applications in effective equations we note that the approximation might be considered more realistic than the exact eigenvalue expression because those equations would be based on semiclassical states. Since such states cannot be eigenstates of the triad but must only be peaked on a certain expectation value, they will automatically give rise to a smearing-out of the spikes in the eigenvalues as discussed in more detail in Sec. 4.1,

\subsubsection{Asymptotic behavior}

This class of correction functions parameterized by two ambiguity parameters $r$ and $j$ captures the most important general properties of such functions, including the position of their maxima at $\tilde{\mu} \approx 1$ (or $\mu \approx j$ ) and the initial power law increase for small $\mu$ (determined by $r$ ) [27, 28]. It is indeed easy to see that all correction functions have the correct classical limit on large scales, such as

$$
\alpha^{(r, j)}(\tilde{\mu}) \approx 1+\frac{1}{32 \tilde{\mu}^{2}} \frac{(r-2)(r-4)}{3} \frac{4\left(3 j^{2}+3 j-1\right)}{5}+O\left(\tilde{\mu}^{-4}\right) \rightarrow 1
$$

for (32). Moreover, for small $\mu$ the correction function goes to zero as

$$
\alpha^{(r, j)}(\tilde{\mu}) \approx(2 \tilde{\mu})^{2-\frac{r}{2}}
$$

which ensures boundedness of the quantized co-triad $e_{(K)} \propto \alpha \sqrt{\tilde{\mu}} \propto \tilde{\mu}^{2}$ (using $r=1$ for this case as in (13)), when $\tilde{\mu} \rightarrow 0$. The same is true for higher $j$ since evaluated at $\mu=0$ the sum of odd terms gives zero. 
This function is not smooth but has a cusp at its maximum at $\mu=1 / 2$, or more generally a cusp at integer or half-integer values between 0 and $j$. The second derivative $\alpha^{\prime \prime}$ is always positive while $\alpha^{\prime}$ changes sign between any two cusps. To the right of the cusp at the largest $\mu$, the derivatives satisfy

$$
\alpha^{\prime}<0, \quad \alpha^{\prime \prime}>0 .
$$

Note that the approximation used for larger $j$ smears out the cusps and does not everywhere have positive second derivative. The behavior above the peak and the general increase below is, however, reproduced well by the approximation. The definite sign of $\alpha^{\prime \prime}$ has farreaching implications in the quantum corrected equations of motion [6]. Small corrections then add up during long cosmic evolution times which would not be realized if, e.g., $\alpha$ would oscillate around the classical value which is also conceivable a priori.

\subsubsection{Small-scale behavior and ambiguities}

We will mostly use here and in cosmological applications of the corrected perturbation equations of [5] the behavior for larger values of $\mu$ above the peak. On very small scales, the approach to zero at $\mu=0$ is special to operators with $\mathrm{U}(1)$-holonomies as they appear in the perturbative treatment here. In particular, as we have seen explicitly the volume operator $\hat{V}$ and gauge covariant combinations of commutators such as $\operatorname{tr}\left(\tau^{i} h\left[h^{-1}, \hat{V}\right]\right)$ commute. It is thus meaningful to speak of the (eigen-)value of inverse volume on zero volume eigenstates. For non-Abelian holonomies such as those for $\mathrm{SU}(2)$ in the full theory, the operators become non-commuting [29]. The inverse volume at zero volume eigenstates thus becomes unsharp and one can at most make statements about expectation values rather than eigenvalues which again requires more information on semiclassical states. Then, the expectation values are not expected to become sharply zero at zero volume, as calculations indeed show [30]. In addition, also here quantization ambiguities matter: We can write volume itself, and not just inverse volume, through Poisson brackets such as [29]

$$
\begin{aligned}
V=\int \mathrm{d}^{3} x( & \frac{\epsilon^{a b c} \epsilon_{i j k}}{6(10 \pi \gamma G / 3)^{3}} \int \mathrm{d}^{3} y_{1}\left\{A_{a}^{i}(x),\left|\operatorname{det} e\left(y_{1}\right)\right|^{5 / 6}\right\} \\
& \left.\times \int \mathrm{d}^{3} y_{2}\left\{A_{b}^{j}(x),\left|\operatorname{det} e\left(y_{2}\right)\right|^{5 / 6}\right\} \int \mathrm{d}^{3} y_{3}\left\{A_{c}^{j}(x),\left|\operatorname{det} e\left(y_{3}\right)\right|^{5 / 6}\right\}\right)^{2} .
\end{aligned}
$$

After a lattice regularization as before, using $V_{v} \approx \ell_{0}^{3}\left|\operatorname{det}\left(e_{a}^{i}\right)\right|$, we obtain

$$
\begin{aligned}
V_{v} & =\ell_{0}^{6}\left(\frac{|\operatorname{det} e|}{\sqrt{V_{v}}}\right)^{2}=\ell_{0}^{6}\left(\epsilon^{a b c} \epsilon_{i j k} \frac{e_{a}^{i}}{V_{v}^{1 / 6}} \frac{e_{b}^{j}}{V_{v}^{1 / 6}} \frac{e_{c}^{k}}{V_{v}^{1 / 6}}\right)^{2} \\
& =\left(\frac{\epsilon^{a b c} \epsilon_{i j k}}{6(10 \pi \gamma G / 3)^{3}} \ell_{0}^{3}\left\{A_{a}^{i}, V_{v}^{5 / 6}\right\}\left\{A_{b}^{j}, V_{v}^{5 / 6}\right\}\left\{A_{c}^{j}, V_{v}^{5 / 6}\right\}\right)^{2}
\end{aligned}
$$

whose quantization, making use of commutators, differs from the original volume operator (12). If non-Abelian holonomies are used, it would not commute with the full volume 
operator of [31] or 32]. This clearly shows that the usual quantization ambiguity also applies to what is considered the relevant geometrical volume. (Related ambiguities for flux operators have been discussed in [21].) It is not necessarily the original volume operator constructed directly from fluxes, but could be any operator having volume as the classical limit. For finding zero volume states to be related to classical singularities, for instance, dynamics indicates that volume constructed in the more complicated way through commutators with the original volume operator is more relevant than the volume operator constructed directly from fluxes [29]. Thus, specific volume eigenstates have to be used with great care in applications with non-Abelian holonomies. Also, the behavior of correction functions below the peak value, especially whether or not they approach zero at zero volume, is thus less clear in a general context. In any case, below the peak positions scales are usually so small, unless one uses larger $j$, that perturbation theory breaks down. The behavior above the peak, by contrast, is robust and gives characteristic modifications to the cosmological evolution of structure.

\section{Effective Hamiltonian}

Calculations of distinct terms in the constraint presented in the preceding section can now be used to derive effective Hamiltonian constraints.

\subsection{Expectation values in semiclassical states and quantum vari- ables}

The derivation of an effective Hamiltonian constraint proceeds by computing expectation values of the constraint operator in semiclassical states which are superpositions of our lattice states peaked on perturbative metric and extrinsic curvature components. Such states are easily constructible although, for the order we are working at here, we do not need to do so explicitly. The peak values of perturbative fields are thus in particular diagonal which means that expectation values can easily be computed via Abelian calculations 5 The only complication arises from the fact that we are necessarily dealing with operators as products of holonomies and fluxes which are not simultaneously diagonalizable. It is most convenient to use the triad eigenbasis $\left|\ldots, \mu_{v, I}, \ldots\right\rangle$ for triad or inverse triad operators, and a holonomy eigenbasis for products of holonomies. This was implicitly assumed previously in the curvature expansion and when using inverse triad eigenvalues for correction functions. However, for expectation values of the complete constraint operator as a product of holonomy and co-triad terms we need to transform between the two eigenbases, which as usually

\footnotetext{
${ }^{5}$ Although initially $\mathrm{SU}(2)$-holonomies appear in the constraint, they only refer to lattice directions such that those holonomies are of the form $\exp \left(A \tau_{I}\right)$. While these matrices do not commute among themselves for different $I$, one can easily re-arrange the order; see, e.g., 33, 33 for a discussion of the analogous effect in symmetric models. The special form of $\mathrm{SU}(2)$-matrices occurring in our context is also the reason why we can take the trace in the Hamiltonian constraint explicitly and reduce it to a product of sines and cosines of curvature components.
} 
is possible by inserting sums over complete sets of states: $\langle\psi|\hat{H}| \psi\rangle=\sum_{I}\left\langle\left|\psi \hat{H}_{1}\right| I\right\rangle\left\langle I\left|\hat{H}_{2}\right| \psi\right\rangle$ if $\{|I\rangle\}$ is the complete set of states and $\hat{H}=\hat{H}_{1} \hat{H}_{2}$ is factorized into the two parts mentioned above. For a complete treatment we thus need to compute matrix elements of $\hat{H}_{1}$ and $\hat{H}_{2}$, not just eigenvalues.

Nevertheless, the calculations presented before already provide the main terms under the following approximation: We assume, without loss of generality, that the complete set of states $\{|I\rangle\}$ contains a state $|\psi\rangle$ we are interested in. More crucially, we assume that the spread $\sigma$ of $\psi$ in basic variables is small. Under this assumption, $\left\langle I\left|\hat{H}_{i}\right| \psi\right\rangle, i=1,2$, are dominated by $\left\langle\psi\left|\hat{H}_{i}\right| \psi\right\rangle$ since (i) there is not much overlap with most other states in the complete set and (ii) the states $|\psi\rangle$, having small spreads, are as close as possible to eigenstates of $\hat{H}_{1}$ and $\hat{H}_{2}$, respectively. With $\hat{H}_{1}$ being a product of holonomies and $\hat{H}_{2}$ depending on fluxes, the spreads required in this construction cannot be arbitrarily small because they are restricted by uncertainty relations. This implies that additional corrections not computed before arise due to the unavoidable spread of semiclassical states. As a direct consequence of spreading, such terms depend on parameters such as $\sigma$ which are nothing but the quantum variables (3) mentioned before. These variables necessarily feature in a complete effective Hamiltonian, describing how spreading and deformations of the state back-react on the peak position [13].

Apart from these quantum variable terms, the main effective Hamiltonian then is of the form $\left\langle\psi\left|\hat{H}_{1}\right| \psi\right\rangle\left\langle\psi\left|\hat{H}_{2}\right| \psi\right\rangle$ where $|\psi\rangle$ is a semiclassical state peaked on a given classical geometry. Each of the two factors is of the form

$$
\sum_{\mu} O_{\mu}\left|\psi_{\mu}^{\left(\mu_{0}\right)}\right|^{2} \sim \sum_{\mu} O_{\mu} f\left(\mu-\mu_{0}\right) \sim \int \mathrm{d} \mu O(\mu) f\left(\mu-\mu_{0}\right)=(O \star f)\left(\mu_{0}\right)
$$

where $O_{\mu}$ are eigenvalues of an operator $\hat{O}$ and $\mu_{0}$ is the peak value of the state $|\psi\rangle$ in the basic variable $\mu$ whose eigenbasis is used. On the right hand side, we see that the effect of computing an expectation value in a semiclassical state is mainly, to the given order, that eigenvalues appear in a form convoluted with the shape of the semiclassical state.

In such a convolution, sharp features in eigenvalue functions such as the spikes in Fig. 5 will be smeared out. But otherwise the general behavior is already displayed well by explicit eigenvalues, and, similarly, higher order curvature corrections are close to what we computed before. For general features we can thus avoid dealing with details of states and their convolution with eigenvalues. The form of effective Hamiltonians we arrive at is thus

$$
\begin{aligned}
H_{\mathrm{eff}}[N]= & \frac{1}{8 \pi G} \int_{\Sigma} \mathrm{d}^{3} x N(x) \alpha[p]\left(-3 \tilde{k}^{2}+\Delta_{K}\right. \\
& \left.+\beta[p]\left(-\tilde{p}^{-1} \partial^{I} \partial_{I} \tilde{p}+\frac{1}{2} \tilde{p}^{-2}\left(\partial^{I} \tilde{p}\right)\left(\partial_{I} \tilde{p}\right)\right)+\Delta_{\partial}^{(1)}\right) \sqrt{|\tilde{p}|} \\
& +\int_{\Sigma} \mathrm{d}^{3} x N(x)\left(\frac{D[p]}{2|\tilde{p}|^{3 / 2}} \pi^{2}+\frac{\sigma[p]|\tilde{p}|^{\frac{1}{2}}}{2}\left(\partial^{I} \phi\right)\left(\partial_{I} \phi\right)+\Delta_{\partial}^{(2)}+|\tilde{p}|^{\frac{3}{2}} U(\phi)\right) \\
& +\int_{\Sigma} \mathrm{d}^{3} x N(x) \Delta_{Q}
\end{aligned}
$$


for metrics including scalar perturbations in longitudinal gauge. Note that the correction functions depend functionally on the field $p(x)$, not $\tilde{p}$, which shows that their scale is uniquely determined by the state irrespective of choices of coordinates. Unspecified correction terms are higher order curvature corrections $\Delta_{K}$ (see Eq. (29)), discretization

corrections $\Delta_{\partial}^{(1 / 2)}$ from different spatial derivative terms in the constraint, and terms containing quantum variables $\Delta_{Q}$ which arise from metric as well as matter fields.

This form of effective constraints also demonstrates potential effects of using $\mathrm{SU}(2)$ representations different from the fundamental one. Notice that we did not compute this for the higher curvature expansion since the required traces of different Pauli matrices are more involved. But it is clear that this can only change the coefficients in the expansion $\Delta_{K}$ since it always remains at a perturbative level. Generally, larger values of $j$ mean that curvature corrections will become important at smaller curvatures compared to $j=1 / 2$, and thus coefficients in an expansion will increase with $j$. The effect in inverse triad corrections $\alpha[p]$, which we did compute explicitly here, is more pronounced since $j$ determines the scale at which one enters the non-perturbative regime of such inverse triad corrections. The main difference between larger values of $j$ and the minimal one is that in the former case peaked states exist whose spread is smaller than the peak position of eigenvalues of an inverse triad operator. When this is realized, the non-perturbative branch of increasing behavior between $\mu=0$ and $\mu=j$ is not completely washed out in the convolution but remains visible in an effective Hamiltonian.

In an effective Hamiltonian this consequence is obvious, but it was not always clear from the underlying difference equation in isotropic models. There, the discrete stepsize, determined by the $\mathrm{SU}(2)$-representation of holonomies in the constraint, equals the peak position of inverse triad eigenvalues (see, e.g., [34]). Thus, the discrete step in the difference equation always jumps from zero directly to the peak when the same representation is used for all holonomies occurring in the gravitational as well as matter parts of the constraint. One could thus argue that dynamics will be insensitive to the value of the representation. Effective equations, if they are applicable in this small-scale regime, show that this is not necessarily so. The representation enters higher curvature terms differently from inverse triad corrections, thus allowing effects of the non-perturbative part to remain.

\subsection{Technical issues}

We now illustrate some of the more important choices we made in constructing the constraint operators used here.

\subsubsection{Quantization procedures}

Our construction is suitable for a treatment of cosmological perturbations within loop quantum gravity, but it does circumvent some of its general aspects. First, we do not take into account full non-abelian features; they can be included perturbatively but are not required for our selection of mode and gauge. Secondly, we do not allow irregular lattices or valence higher than six. Also this can be included by summing operator contributions 
over triples of edges (as they are constructed in the full setting). Detailed coefficients in effective expressions may then change, but not qualitative effects. Moreover, as already mentioned the labels coming with additional edges or higher valent vertices are redundant for cosmological perturbations.

We have presented higher power corrections using "holonomies" based on extrinsic curvature rather than connection components since this simplifies the calculations considerably. Using the background, it is mathematically possible to define such objects, although in a full background independent setting only holonomies of a connection would be well-defined. We use this mainly as a first possibility to demonstrate which types of corrections one expects and will discuss now how general the resulting expressions can be considered to be. This refers to corrections to terms of the Lorentzian constraint which, schematically, can be written as

$$
F+K^{2}=\mathrm{d} A+A^{2}+(A-\Gamma)^{2}=\mathrm{d}(\Gamma+K)+(\Gamma+K)^{2}+K^{2}
$$

to be multiplied by triad components dealt with by Poisson brackets.

Using extrinsic curvature as basic object, one obtains trigonometric functions of its components which when expanded give higher power corrections to $\mathrm{d} K+K^{2}$. But since the spin connection has been split off from the basic object, one has to quantize it individually and add suitable combinations for $\mathrm{d} \Gamma+\Gamma^{2}$ to the constraint. Here, we assume that the cross-term $\Gamma K$ does not contribute which is indeed the case for diagonal triads (implying anti-symmetric spin connection components) and extrinsic curvature. This is not much of a restriction: $K$ is required to be diagonal for $K$-holonomies to simplify the calculations. Moreover, the perturbative non-diagonal part of $\Gamma$ must be antisymmetric because it perturbs an $\mathrm{SO}(3)$-matrix. If there is a diagonal contribution, e.g. from a spatially curved background, it can be combined with $K$. As for corrections, we have higher power corrections in the quantization of $\mathrm{d} K+K^{2}$ and inverse triad corrections in $\mathrm{d} \Gamma+\Gamma^{2}$ since the spin connection contains inverse triad components.

Using $A$-holonomies gives, at first sight, a different picture. Now, $F=\mathrm{d} A+A^{2}$ receives higher power corrections, but the spin connection is not quantized directly, not giving immediate inverse triad corrections. One rather has to proceed as in the full theory [18] where the term $(A-\Gamma)^{2}=K^{2}$ in the constraint is re-written using (16). The double-Poisson bracket (16) used to quantize extrinsic curvature now leads to additional corrections. In particular, since inverse triad quantizations have been used in $H^{(1)}$ in (17), corresponding corrections do arise which are qualitatively similar to those in a direct quantization of the spin connection. One thus expects similar types of corrections, as with $K$-holonomies and $\Gamma$-quantizations, although in different combinations.

In our construction, $K$-holonomies arose from $A$-holonomies through a perturbative expansion in non-diagonal components. When using $A$-holonomies on a spatially curved background such as a closed Friedmann-Robertson-Walker model, it is furthermore necessary to take lattice effect of an inhomogeneous model [7] or related effects [35] into account. A fine enough lattice $(\mathcal{N} \gg 1)$ is required for a semiclassical expansion of holonomies since

$$
\exp (i A) \sim \exp (i(\Gamma+K)) \sim \exp \left(i\left(K+\mathcal{N}^{-1 / 3} \bar{\Gamma}+\delta \Gamma\right)\right)
$$


with the background spin connection possibly of the order $\bar{\Gamma}=V_{0}^{1 / 3} \tilde{\bar{\Gamma}}=O(1)$ can be expanded in all terms only if $\mathcal{N}$ is large. (For a closed isotropic model, for instance, $\bar{\Gamma}=1 / 2$ [36].) The number of vertices $\mathcal{N}$ enters through $\ell_{0} \tilde{\bar{\Gamma}}=\mathcal{N}^{-1 / 3} \bar{\Gamma}$ in holonomies. The spin connection perturbation $\delta \Gamma$ will be small in perturbative regimes such that we can always write

$$
\exp \left(i\left(K+\mathcal{N}^{-1 / 3} \bar{\Gamma}+\delta \Gamma\right)\right)=\exp \left(i\left(K+\mathcal{N}^{-1 / 3} \bar{\Gamma}\right)\right)(1+i \delta \Gamma+\cdots)
$$

But the remaining exponential also has to reduce to the leading terms of an expansion in semiclassical regimes. While $K$ is then automatically small, this may not be the case for $\bar{\Gamma}$. Without the reduction by $\mathcal{N}^{-1 / 3}$ for a fine lattice, one could not expand the exponentials to reproduce the polynomials in $K$ and $\Gamma$ classically occurring in the constraints.

We have focused here on the first type of quantization which is simpler to compute explicitly but may not be as close to the full theory. Using $A$-holonomies, no diagonality can be used, but perturbative treatments of non-diagonal components are possible. It is thus possible, though more involved, to compute correction terms obtained through different quantization schemes and to compare their consequences, in particular those at the phenomenological level. A first result in this direction follows from the perturbation equations derived in [5] which show that effects of inverse triad corrections in $\Gamma$ are less significant than those in the commutator [6]. Thus, one can hope that the precise quantization procedure of curvature is not very important for physical aspects accessible so far. A detailed investigation of all consequences can nevertheless provide important guidance as to which procedure should be pursued in the full theory.

\subsubsection{Different types of correction functions}

In fact, we have included in the computation of perturbative effects four different correction functions $\alpha, \beta, D$ and $\sigma$. All of them come from inverse triad corrections. With all functions coming from the same type of modification, one may wonder why they should not all be identical.

It is clear from the procedure that these functions arise from different classical functionals of densitized triad components. For instance, $\alpha$ comes from the antisymmetric part of $E_{i}^{a} E_{i}^{b} / \sqrt{|\operatorname{det} E|}$ while $\sigma$ comes from the symmetric part. They could be related to the same correction, but the quantization requires quite different rewritings (25) and (50) of the corresponding terms in Hamiltonians such that correction functions will differ. In particular, they come with different parameters such as $r$. On top of that, each correction function is subject to quantization ambiguities. As we have seen, however, the typical behavior is robust under changes of the parameters. In particular, all correction functions have the same qualitative properties and differ only quantitatively in a way parameterized by a few parameters. 


\subsubsection{Implications for gauge issues}

The assumptions on states used to derive effective constraints have a bearing on the gauge issue. By specifying the peak value of a spatial geometry and its extrinsic curvature in a semiclassical state we are fixing the spatial diffeomorphism constraint rather than solving it by averaging as done in the full theory [37]. Choosing the form of peak values partially implements a chosen gauge, but still allows some freedom. We also note that even though spatial diffeomorphisms are fixed, one still has to impose the constraint. This will give rise to one of the cosmological perturbation equations as is clear from [5].

Fixing the diffeomorphism constraint also implies a different viewpoint for the Hamiltonian constraint operator of the loop quantization. In the full construction [18], one makes use of diffeomorphisms in order to make the operator more independent of the choice of edges used to quantize curvature. When diffeomorphisms are fixed, this is no longer possible and effective constraints would depend on precisely how such edges are chosen. We have fixed this freedom here by laying the edges entirely on the lattice resulting in a graph preserving operator. Thus, holonomy corrections in the constraint depend on the lattice spacing provided through a state implementing the background geometry. While this simplifies the calculations without leading to significant quantitative changes in coefficients, we are as a consequence disregarding the creation of new vertices by the constraint operator. Thus, $\mathcal{N}$ is constant for the construction, but may effectively be assumed to be slowly dependent on, e.g., the total volume (see [7] for more details).

\section{General implications for effective theory}

Our calculations, following the scheme to derive effective equations sketched before, have led to corrections (64) which arise as leading order terms in an effective theory of perturbative loop quantum gravity. No complete expression has been derived, but characteristic terms are clear and lead to interesting phenomena [6]. Rather than studying one model in detail we have provided here an illustration of the general scheme: The characteristic feature of loop quantizations is the use of holonomies, which give rise to typical correction terms. They can be split into higher power corrections, which are always perturbative, and corrections to inverse powers of triad components which become non-perturbative at small scales. All these corrections are in addition to discretization and genuine quantum effects such as higher time derivatives. In this section we highlight conceptual conclusions that can be drawn from such a scheme.

\subsection{Basic variables in quantum gravity corrections}

Holonomy corrections arise through expectation values and thus depend on the basic variables used in the quantization. Using commutators to quantize triad components, for instance, modifies the classical expressions in a way which can be computed through the explicitly available eigenvalues such as (56) of these operators. The occurrence of trigonometric functions instead of direct curvature or connection components leads to higher power 
terms when expanded in an effective constraint. Such corrections depend, by construction, on $p(x) / \ell_{\mathrm{P}}^{2}=\ell_{0}^{2} \tilde{p}(x) / \ell_{\mathrm{P}}^{2}$ and $k(x)=\ell_{0} \tilde{k}(x)$, respectively, both of which are independent under rescaling the coordinates. They do depend, however, on the lattice size which determines the scales on which a state probes the field.

If we split the fields into background parts

$$
\tilde{\bar{p}}:=\frac{1}{V_{0}} \int \mathrm{d}^{3} x \tilde{p}(x) \quad \text { and } \quad \tilde{\bar{k}}:=\frac{1}{V_{0}} \int \mathrm{d}^{3} x \tilde{k}(x)
$$

by integrating over a cube (sufficiently large to contain, say, the Hubble volume) of coordinate volume $V_{0}=\int \mathrm{d}^{3} x$ and perturbations

$$
\delta \tilde{p}(x)=\tilde{p}(x)-\tilde{\bar{p}} \quad \text { and } \quad \delta \tilde{k}(x)=\tilde{k}(x)-\tilde{\bar{k}}
$$

to set up cosmological perturbation theory [5],6 we can see from preceding constructions that it is not these fields directly which occur in correction functions. In isotropic loop quantum cosmology, the quantization is based on variables

$$
\bar{p}=V_{0}^{2 / 3} \tilde{\bar{p}} \approx \frac{1}{V_{0}^{1 / 3}} \sum_{v} \ell_{0}^{3} \tilde{p}(v)=\frac{1}{\mathcal{N}^{1 / 3}} \sum_{v} p(v)
$$

and

$$
\bar{k}=V_{0}^{1 / 3} \tilde{\bar{k}} \approx \frac{1}{V_{0}^{2 / 3}} \sum_{v} \ell_{0}^{3} \tilde{k}(v)=\frac{1}{\mathcal{N}^{2 / 3}} \sum_{v} k(v)
$$

which now appear as lattice averages in an inhomogeneous setting and provide the background for cosmological perturbation theory. As such, they do not depend on the auxiliary coordinate volume $V_{0}$ as they would in homogeneous models [17] but on the number $\mathcal{N}$ of lattice vertices. These two quantities are related by $V_{0}=\mathcal{N} \ell_{0}^{3}$ through the lattice size $\ell_{0}$, but $\mathcal{N}$ has significance as a parameter specifying the states rather than just being auxiliary as $V_{0}$. Similarly, basic variables of the inhomogeneous theory are function: 7

$$
p(x)=\ell_{0}^{2} \tilde{p}(x)=\frac{\ell_{0}^{2}}{V_{0}^{2 / 3}} \bar{p}+\delta p(x)=\frac{1}{\mathcal{N}} \sum_{v} p(v)+\delta p(x)
$$

which directly occur in correction functions through fluxes, and

$$
k(x)=\ell_{0} \tilde{k}(x)=\frac{\ell_{0}}{V_{0}^{1 / 3}} \bar{k}+\delta k(x)=\frac{1}{\mathcal{N}} \sum_{v} k(v)+\delta k(x)
$$

occurring in higher power corrections through holonomies.

\footnotetext{
${ }^{6}$ In [5], only written here with a tilde have been used, but the tilde was dropped for notational convenience.

${ }^{7}$ Note that $\ell_{0}$ is used to rescale the inhomogeneous $\tilde{p}(x)$ while $V_{0}$ is used to rescale $\tilde{\bar{p}}$ as it is done in isotropic models [17.
} 
This shows that the resulting equations are rescaling invariant when $\tilde{p}, \tilde{k}$ and $\ell_{0}$ change simultaneously, a fact which was not always obvious in isotropic models based on the scale factor. As expected, the equations are also dependent on specifics (mainly $\mathcal{N}$ ) of the state whose dynamics is described effectively. This shows which states are suitable for perturbation theory and when perturbations break down. A perturbation scheme works only if $\delta \tilde{p} \ll \tilde{\bar{p}}$ which from (170) implies that differences between local edge labels of the state (corresponding to $\delta p(x)=\ell_{0}^{2} \delta \tilde{p}(x)$ ) must be small compared to the average lattice label $\mathcal{N}^{-1} \sum_{v} p(v)$ (corresponding to the perturbative background value of $p(x)$ ). Since the labels are discrete, differences between them have a positive lower bound unless they are equal. Thus, the average label must be large compared to the discrete gap in the spectrum of labels. In our U(1)-theory, labels are integer valued which means that the average label must be larger than one, and local edge labels must not stray too much from the average. There is no such restriction from the curvature perturbations because curvature does not have a discrete spectrum.

\subsection{Quantum variables and classical limit}

Starting from the Hamiltonian (constraint) operator in any quantum theory, the quantum Hamiltonian is defined as a function on the projective Hilbert space determined by taking expectation values. This can be seen as the Hamiltonian function of a dynamical system whose phase space is obtained from the Hilbert space [38, 39, 40]. The system thus appears of classical form at least as far as dynamics is concerned, but each of its classical degrees of freedom is accompanied by infinitely many quantum variables (3). An effective description requires a further step, truncating the infinitely many quantum variables to a finite set [12. If this is done consistently, one obtains effective equations which amend the classical ones by quantum corrections. One often performs such a truncation by using a certain class of semiclassical states to compute expectation values of the Hamiltonian operator. The regime under consideration determines what a suitable set of semiclassical states is.

Based on the assumed semiclassicality of states peaked at values $p^{I}$ and $k_{J}$, the expressions we derived give the main part of the effective Hamiltonian constraint computed as an expectation value in such states. Note that we did not explicitly compute expectation values in states but read off corrections from operators by expanding trigonometric functions arising from holonomies or eigenvalues of inverse triad operators. Each of these corrections requires, strictly speaking, eigenstates of holonomies for higher order corrections in $\tilde{k}$ or flux eigenstates for corrections as functions of $\tilde{p}$. But even if we were to compute expectation values in peaked states, the main corrections would be of the form read off from different eigenstates as seen by analogous calculations in the isotropic case [17, 41]. In general, one has to use semiclassical states which are neither eigenstates of holonomies nor of flux operators. This gives rise to additional contributions depending, e.g., on the spread of the state. From the spread and other detailed properties of states one obtains contributions depending on additional independent quantum variables corresponding to fluctuations and correlations. For non-quadratic Hamiltonians or constraints, these quantum variables couple to classical variables and influence their motion. To some degree, 
the appearance of additional independent quantum variables corresponds to higher derivative terms in effective actions [13]. Thus, we obtain modified coefficients (from correction functions such as $\alpha$ ), higher powers in momenta (from $\sin k_{I}$ and $\cos k_{I}$ ) and higher derivative terms from quantum variables (interpreted as higher time derivatives) and from the discretization (higher spatial derivatives), which comprise all effects known and expected from effective actions. The first two arise as typical corrections by using holonomies.

\subsubsection{Basic variables vs. coarse graining}

In our treatment here we assumed that lattice scales are small compared to other scales of the relevant physical fields such as matter or classical metric modes to be obtained in a semiclassical limit. In such a context, it is sufficient to use the basic variables as they come as labels of a quantum state directly in effective correction functions. This is not possible in regimes where basic variables of the states are themselves strongly inhomogeneous as it necessarily happens when the discrete flux labels $\mu_{v, I}$ approach the lowest non-zero value one. Then, the perturbative condition $\delta \mu_{v, I} \ll \mu_{v, I}$ where $\delta \mu_{v, I}$ refers to the difference in nearby labels cannot be satisfied unless $\delta \mu_{v, I}=0$, i.e. the labels are exactly homogeneous. Most likely, this happens in strong curvature regimes where perturbation theory would be expected to break down even classically. But since the discreteness of the labels $\mu_{v, I}$ plays a role in this simple argument, there can be regimes where classical perturbation theory would be applicable but the underlying lattice formulation would not seem to be in a perturbative regime. In such cases, one would have to coarse grain the basic variable, i.e. replace the basic lattice site variables by averages over larger patches of an intermediate scale. Then, the averaged labels would increase, relieving the contradiction between $\delta \mu_{v, I} \ll \mu_{v, I}$ and quantum discreteness.

\subsubsection{Orders of magnitude of corrections}

With several different correction terms, it is helpful to know whether in certain regimes some of them can be ignored. This can be difficult to determine in homogeneous models unless one makes special choices of ambiguity parameters such as large values of $j$ [42. In inhomogeneous situations it is often simpler to determine which corrections are expected to be dominant because they depend differently on the basic scale contained in $p_{v, I}$ [7]. These variables are parameters determining the state and thus the physical regime being probed. When $p_{v, I}$ is small, i.e. close to its minimum $\ell_{\mathrm{P}}^{2}$, inverse triad corrections are large. They decrease when $p_{v, I}$ becomes larger, but this also implies larger and fewer lattice sites such that discretization effects become important. Moreover, in nearly isotropic configurations extrinsic curvature is given by

$$
k_{v, I}=\sqrt{8 \pi G p_{v, I} \rho / 3}
$$

as it follows from the Friedmann equation. The energy density scale $\rho$ thus determines when curvature corrections are relevant. Since there is also a factor of $p_{v, I}$, curvature corrections increase with larger $p_{v, I}$ just as discretization corrections. 
For a semiclassical regime we must have $p_{v, I}>\ell_{\mathrm{P}}^{2}$ in order to reproduce closely the correct inverse powers of triad components. We must also have a discreteness scale $p_{v, I}$ which is sufficiently small in order to avoid discretization effects already in, say, particle physics. This requires $p_{v, I}$ to be much smaller than the typical physical scale squared, such as a wave length $\lambda$ of field modes or even the Hubble length $a / \dot{a}$. We thus have a range $\ell_{\mathrm{P}}^{2}<p_{v, I} \ll \lambda^{2}$ or $\ell_{\mathrm{P}}^{2}<p_{v, I} \ll(8 \pi G \rho)^{-1}$ if we express the Hubble length in terms of energy density. At the upper bound we ensure that discretization effects do not disrupt other physics used essentially in a given scenario. As a consequence of (72), this implies that higher order corrections in curvature are small, too. The dominant contributions are then given by inverse triad corrections which we have focused on in the preceding derivations. Note that the semiclassical range for $p_{v, I}$ is large in late time cosmology, implying that corrections can be arbitarily small, for instance to the propagation of signals from gamma ray bursts. In the early universe, however, and in particular during inflation the energy scale is much higher, restricting the range more narrowly [6]. The best tests of quantum gravity effects are thus expected from early universe cosmology.

\subsubsection{Classical limit}

We have ignored in our calculations so far any detailed specifics of states and terms containing quantum variables. Implicitly, we are thus assuming that such terms are subdominant, just as one assumes analogous terms to be subdominant in a derivative expansion of low energy effective actions. Under this assumption we reproduce classical expressions in the suitable limit, which proves that loop quantum gravity has the correct classical limit in this perturbative regime in the same sense as in usual effective theories. This statement certainly includes inhomogeneities in the perturbative sector considered here. For instance, the Newton potential and corrections on smaller scales can be obtained from perturbation equations derived from the effective constraints [6].

In effective theory, verifying the correct classical limit does not require one to construct explicit dynamical coherent states, not even approximately. This would certainly be of interest, but would be highly complicated and is rarely done in interacting field theories where one can nevertheless be certain about the correct classical limit. We emphasize that, in any case, a discussion of the semiclassical limit based on coherent states does require such states to be dynamical coherent states, or at least must involve statements on dynamical changes of state parameters to specify suitable regimes. This means that states must stay approximately coherent under evolution or, in a fully constrained theory such as gravity, solve the Hamiltonian constraint. If this is not realized, quantum variables and the back-reaction of spread and deformations on the classical variables are not under sufficient control to ensure the correct classical limit.

There are two viable procedures to verify the correct classical limit of a quantum theory, be it a constrained or unconstrained system. First, one may use kinematical coherent states to compute expectation values of the dynamical operators (a Hamiltonian or constraint operators) and then analyze the dependence of quantum variables in resulting equations of motion; if their effect on expectation values is small in suitable regimes, the 
correct classical limit results. Secondly, dynamical coherent states can be used if they can be constructed at least approximately, which directly illustrates whether the dynamics of expectation values is close to the classical one. The second procedure is much more complicated for interacting theories since the full quantum dynamics would have to be solved at least approximately at the quantum level. The first procedure, by contrast, allows one to derive effective dynamical equations first and then approximate solutions to understand the behavior of expectation values. Thus, usually kinematical semiclassical states are used in explicit effective descriptions, followed by an analysis such as one in a derivative expansion in quantum field theory. Such a further analysis is always required when kinematical semiclassical states are used, and it can only be done in a regime dependent way to bring in conditions for when semiclassicality should be satisfied. We have done this implicitly in our discussion by assuming slowly varying fields as in usual derivative expansions, both in space by doing a continuum limit of the lattice states and in time by assuming quantum variables to be negligible.

We emphasize again that even if one can demonstrate an "instant" classical limit by using kinematical coherent states, a dynamical statement would still require one to assume (or to show) that such back-reaction effects of quantum variables on expectation values are not strong. This picks the correct regime of states in which one has semiclassical behavior. Without such an additional analysis, kinematical coherent states would neglect the backreaction of spreading and deformations of states on expectation values which are essential for dynamical effective equations [12]. An additional aspect arises for generally covariant situations where not all variables can be peaked in a semiclassical state as it would be the case in a common kinematical coherent state. Some of the phase space variables will have to play the role of internal "clocks" in which evolution of expectation values as well as quantum variables is measured. Thus, when constructing kinematical coherent states to check the classical limit, they must not be peaked on all phase space variables; a choice of clock has to be made before the calculation. Then, quantum variables also back-react on the change of the clock.

Often, investigations of classical limits based on kinematical coherent states are motivated by well-known constructions of the harmonic oscillator or free quantum field theories. The behavior of quantum variables or of dynamical coherent states in general can, however, be very different from the well-studied aspects of the free systems. Such systems or small deviations from them with anharmonicity can well be studied by coherent state techniques. But gravity is very different and not expanded around a set of harmonic oscillators. In fact, gravity with its unbounded Hamiltonian even lacks a ground state or vacuum to expand around. The bounce model solved in [20], for instance, shows that the spreads change exponentially rather than being constant or at least periodic as it happens for the harmonic oscillator. The resulting semiclassical picture is very different from that provided by harmonic oscillator coherent states. This must be taken into account in semiclassical analyses; effective theory provides suitable means to study such situations in sufficiently general terms as initiated in this paper. 


\subsection{Collective graviton}

The constructions indicate a picture of the classical limit of quantum gravity where linear metric modes appearing in the evolution equations are not basic excitations of a quantum field. They arise, rather, as collective excitations out of the underlying discrete quantum theory. At a basic level, degrees of freedom are encoded in quantum numbers $\mu_{v, I}$ while the scalar mode, for instance, is obtained through the difference between such a local label and the average value on the whole lattice. The classical modes thus arise as non-local, effective excitations out of the underlying quantum state [7]. This shows in a well-defined sense how classical degrees of freedom are obtained as collective excitations, analogously to phonons in a crystal. That the correct classical dynamics results for these collective modes is demonstrated, for instance, by the derivation of Newton's potential for perturbations on a flat isotropic background in [6].

\section{Summary}

Together with [12, 7, 5] we have shown in this paper that techniques are now available to derive effective equations of cosmological perturbation theory. The geometrical background on which cosmological perturbations are defined is introduced through a class of states, rather than being used to set up the quantization. Background independent quantum properties thus remain, but one can make use of perturbation expansions for explicit calculations. The role of quantum labels and basic variables is clear from this procedure, which determines the type as well as order of magnitudes of correction terms which remained obscure previously. The inhomogeneous treatment including all relevant modes allows us to see all possible correction terms. Note, for instance, that since the isotropic expression for the co-triad is finite on small scales even classically, it would not contribute a correction function to the gravitational part of the Hamiltonian constraint in a purely isotropic setting. When isotropic expressions are quantized directly, a cancellation of the inverse isotropic triad component hides possible quantum effects of the full constraint. This does not arise in our context starting from an inhomogeneous lattice quantization. Thus, complete corrections are obtained in reliable form.

Many different regimes are still to be explored to obtain a full overview of all effects. Moreover, gauge issues have to be investigated which is of relevance for the full quantum theory, too. While general effective equations including the relevant inhomogeneities are now available and orders of correction terms can be estimated, one still has to use them with care since they are not yet formulated for gauge invariant perturbations. In this context one should notice that not only evolution equations but also gauge transformations are determined by the constraints and thus modified by quantum corrections. It is thus not possible to use classical expressions for gauge invariant quantities since they will receive additional corrections. These issues are currently being studied to complete the derivation of equations with quantum corrections. The strategy for computing those corrections from quantum operators has been provided in this paper. 
When speaking of quantum corrections to classical equations it is clear that to zeroth order the correct classical limit has to be satisfied. In fact, what we have shown here implies that loop quantum gravity has the correct classical limit for scalar modes in longitudinal gauge propagating on a spatially flat background. In the process, we have demonstrated which steps must be involved in such a detailed calculation, most importantly a continuum limit but also a slowly varying field approximation. Extensions to other modes and gauges, and different backgrounds, can be done by the same techniques but are technically more involved to do explicitly. Nevertheless, it is clear from the construction that the correct classical limits will also be reproduced in those cases. More precisely, we have shown in Sec. 5.2.2 that there are always ranges of the basic lattice variables such that quantum corrections are small in nearly classical situations of low energy density and small curvature. In more energetic cosmological situations, those ranges can shrink to narrow intervals such that significant quantum corrections can be expected [6].

This demonstration of the correct classical limit crucially rests on a new understanding of effective theory [12]. Although it has not yet been formulated fully for field theories (but see [14]), this scheme is applicable here due to the ultraviolet cut-off of quantum gravity. On any lattice state we have only finitely many degrees of freedom in any compact spatial volume to which the quantum mechanical techniques of [12] directly apply. While loop quantum gravity does not possess a sharp cut-off but is rather based on arbitrary graphs in space any of which can occur in a general superposition [37], effective equations are always defined with respect to a single class of states. The physical state thus determines the cut-off dynamically. We have certainly not used explicit physical solutions of the Hamiltonian constraint but rather computed effective equations from general states. If a physical solution were available, the labels $p_{v}$ would be determined explicitly and fix the order of correction terms completely. Moreover, a full solution would determine how the lattice itself changes by the creation of new vertices in terms of an internal clock such as the total volume. This graph-changing nature seems to be one of the most important effects to be understood especially for late-time evolution in cosmology, or any dynamical issue relevant for large spatial slices. Although such a full solution seems currently out of reach, models and effective analyses already provide quite detailed information on the dynamics of background independent quantum gravity in cosmologically relevant regimes.

\section{Acknowledgements}

MB was supported by NSF grant PHY-0554771, HH by the fellowship A/04/21572 of Deutscher Akademischer Austauschdienst (DAAD) and MK by the Center for Gravitational Wave Physics under NSF grant PHY-01-14375. We thank Parampreet Singh for joining us in initial calculations of this project. 


\section{References}

[1] M. Bojowald, Isotropic Loop Quantum Cosmology, Class. Quantum Grav. 19 (2002) 2717-2741, gr-qc/0202077

[2] M. Bojowald, Homogeneous loop quantum cosmology, Class. Quantum Grav. 20 (2003) 2595-2615, gr-qc/0303073

[3] M. Bojowald, Spherically Symmetric Quantum Geometry: States and Basic Operators, Class. Quantum Grav. 21 (2004) 3733-3753, gr-qc/0407017]

[4] M. Bojowald, Loop Quantum Cosmology, Living Rev. Relativity 8 (2005) 11, gr-qc/0601085], http://relativity.livingreviews.org/Articles/lrr-2005-11/

[5] M. Bojowald, H. Hernández, M. Kagan, P. Singh, and A. Skirzewski, Hamiltonian cosmological perturbation theory with loop quantum gravity corrections, [gr-qc/0609057]

[6] M. Bojowald, H. Hernández, M. Kagan, P. Singh, and A. Skirzewski, Formation and evolution of structure in loop cosmology, astro-ph/0611685]

[7] M. Bojowald, Loop quantum cosmology and inhomogeneities, Gen. Rel. Grav., to appear, gr-qc/0609034

[8] R. Arnowitt, S. Deser, and C. W. Misner, The Dynamics of General Relativity, Wiley, New York, 1962

[9] A. Ashtekar, New Hamiltonian Formulation of General Relativity, Phys. Rev. D 36 (1987) 1587-1602

[10] J. F. Barbero G., Real Ashtekar Variables for Lorentzian Signature Space-Times, Phys. Rev. D 51 (1995) 5507-5510, |gr-qc/9410014

[11] G. Immirzi, Real and Complex Connections for Canonical Gravity, Class. Quantum Grav. 14 (1997) L177-L181

[12] M. Bojowald and A. Skirzewski, Effective Equations of Motion for Quantum Systems, Rev. Math. Phys. 18 (2006) 713-745, math-ph/0511043

[13] M. Bojowald and A. Skirzewski, Quantum Gravity and Higher Curvature Actions, In Current Mathematical Topics in Gravitation and Cosmology (42nd Karpacz Winter School of Theoretical Physics), [hep-th/0606232]

[14] A. Skirzewski, Effective Equations of Motion for Quantum Systems, PhD thesis, Humboldt-Universität Berlin, 2006

[15] C. Rovelli and L. Smolin, Loop Space Representation of Quantum General Relativity, Nucl. Phys. B 331 (1990) 80-152 
[16] C. Rovelli and L. Smolin, Spin Networks and Quantum Gravity, Phys. Rev. D 52 (1995) 5743-5759

[17] A. Ashtekar, M. Bojowald, and J. Lewandowski, Mathematical structure of loop quantum cosmology, Adv. Theor. Math. Phys. 7 (2003) 233-268, gr-qc/0304074

[18] T. Thiemann, Quantum Spin Dynamics (QSD), Class. Quantum Grav. 15 (1998) 839-873, gr-qc/9606089

[19] C. Rovelli and L. Smolin, The physical Hamiltonian in nonperturbative quantum gravity, Phys. Rev. Lett. 72 (1994) 446-449, gr-qc/9308002

[20] M. Bojowald, Large scale effective theory for cosmological bounces, gr-qc/0608100

[21] K. Giesel and T. Thiemann, Consistency Check on Volume and Triad Operator Quantisation in Loop Quantum Gravity I, Class. Quantum Grav. 23 (2006) 5667-5691, gr-qc/0507036

[22] A. Ashtekar, J. Lewandowski, and H. Sahlmann, Polymer and Fock representations for a Scalar field, Class. Quantum Grav. 20 (2003) L11-L21, gr-qc/0211012

[23] T. Thiemann, QSD V: Quantum Gravity as the Natural Regulator of Matter Quantum Field Theories, Class. Quantum Grav. 15 (1998) 1281-1314, [gr-qc/9705019]

[24] H. Sahlmann and T. Thiemann, Towards the QFT on Curved Spacetime Limit of QGR. I: A General Scheme, Class. Quantum Grav. 23 (2006) 867-908, gr-qc/0207030]

[25] M. Bojowald, J. E. Lidsey, D. J. Mulryne, P. Singh, and R. Tavakol, Inflationary Cosmology and Quantization Ambiguities in Semi-Classical Loop Quantum Gravity, Phys. Rev. D 70 (2004) 043530, gr-qc/0403106

[26] M. Gaul and C. Rovelli, A generalized Hamiltonian Constraint Operator in Loop Quantum Gravity and its simplest Euclidean Matrix Elements, Class. Quantum Grav. 18 (2001) 1593-1624, gr-qc/0011106

[27] M. Bojowald, Quantization ambiguities in isotropic quantum geometry, Class. Quantum Grav. 19 (2002) 5113-5130, gr-qc/0206053]

[28] M. Bojowald, Loop Quantum Cosmology: Recent Progress, In Proceedings of the International Conference on Gravitation and Cosmology (ICGC 2004), Cochin, India, Pramana 63 (2004) 765-776, gr-qc/0402053

[29] M. Bojowald, Degenerate Configurations, Singularities and the Non-Abelian Nature of Loop Quantum Gravity, Class. Quantum Grav. 23 (2006) 987-1008, gr-qc/0508118

[30] J. Brunnemann and T. Thiemann, Unboundedness of Triad-Like Operators in Loop Quantum Gravity, Class. Quantum Grav. 23 (2006) 1429-1483, gr-qc/0505033 
[31] C. Rovelli and L. Smolin, Discreteness of Area and Volume in Quantum Gravity, Nucl. Phys. B 442 (1995) 593-619, gr-qc/9411005], Erratum: Nucl. Phys. B 456 (1995) 753

[32] A. Ashtekar and J. Lewandowski, Quantum Theory of Geometry II: Volume Operators, Adv. Theor. Math. Phys. 1 (1997) 388-429, gr-qc/9711031

[33] M. Bojowald, Loop Quantum Cosmology: I. Kinematics, Class. Quantum Grav. 17 (2000) 1489-1508, gr-qc/9910103

[34] K. Vandersloot, On the Hamiltonian Constraint of Loop Quantum Cosmology, Phys. Rev. D 71 (2005) 103506, gr-qc/0502082

[35] A. Ashtekar, T. Pawlowski, and P. Singh, Quantum Nature of the Big Bang: An Analytical and Numerical Investigation, Phys. Rev. D 73 (2006) 124038, [gr-qc/0604013]

[36] M. Bojowald and K. Vandersloot, Loop quantum cosmology, boundary proposals, and inflation, Phys. Rev. D 67 (2003) 124023, gr-qc/0303072

[37] A. Ashtekar, J. Lewandowski, D. Marolf, J. Mourão, and T. Thiemann, Quantization of Diffeomorphism Invariant Theories of Connections with Local Degrees of Freedom, J. Math. Phys. 36 (1995) 6456-6493, gr-qc/9504018

[38] T. W. B. Kibble, Geometrization of quantum mechanics, Commun. Math. Phys. 65 (1979) 189-201

[39] A. Heslot, Quantum mechanics as a classical theory, Phys. Rev. D 31 (1985) 1341-1348

[40] A. Ashtekar and T. A. Schilling, Geometrical Formulation of Quantum Mechanics, In On Einstein's Path: Essays in Honor of Engelbert Schücking, Ed A. Harvey, p. 23-65, Springer, New York, 1999, gr-qc/9706069

[41] J. Willis, On the Low-Energy Ramifications and a Mathematical Extension of Loop Quantum Gravity, PhD thesis, The Pennsylvania State University, 2004

[42] M. Bojowald, Inflation from quantum geometry, Phys. Rev. Lett. 89 (2002) 261301, gr-qc/0206054 